\title{
Theoretical Modeling and Machining Experiments of Cylindrical Microstructure Assisted by Single Point Diamond Turning
}

Jingjin Li

Jilin University

Shijun Ji ( $\nabla$ jishijun@jlu.edu.cn )

Jilin University https://orcid.org/0000-0003-3269-6770

Ji Zhao

Jilin University

Jianfeng Li

Jilin University

Handa Dai

Jilin University

\section{Research Article}

Keywords: Cylindrical sine wave groove micro feature surface (CSWGS), Cylindrical sinusoidal mesh micro feature surface (CSMS), Single point diamond turning, Micro-feature surface modeling, Turning trajectory planning, Error theories

Posted Date: April 16th, 2021

DOI: https://doi.org/10.21203/rs.3.rs-394473/v1

License: (9) (1) This work is licensed under a Creative Commons Attribution 4.0 International License. Read Full License 


\title{
Theoretical Modeling and Machining Experiments of Cylindrical Microstructure assisted by Single Point Diamond Turning
}

\author{
Jingjin Li ${ }^{\text {a }}$, Shijun $\mathrm{Ji}^{*}{ }^{\mathrm{b}}$, Ji Zhao ${ }^{\mathrm{c}}$, Jianfeng Li ${ }^{\mathrm{d}}$, Handa Dai ${ }^{\mathrm{e}}$ \\ School of Mechanical and Aerospace Engineering, Jilin University, China \\ a2636787092@qq.com; jishijun@jlu.edu.cn; ${ }^{\mathrm{c}}$ jzhao@jlu.edu.cn; ${ }^{\mathrm{d}} 1597232496 @ q q . c o m ;{ }^{\mathrm{e}}$ dhd@jlu.edu.cn
}

\begin{abstract}
:
Micro structure requires nanometer-scale surface roughness and micro- or even sub-micron form error accuracy in different applications. Two kinds of modeling theories and methods of micro-feature of rotating body and non-rotating body are studied, and the corresponding tool turning trajectory planning method is put forward. In order to process the designed micro-feature structure successfully and avoid the interference and overcutting between tool and workpiece caused by improper selection of tool parameters, the cutting parameters are analyzed and two error theories are proposed. Then a precision driven turning trajectory planning method is proposed, which can optimize the turning parameters according to the setting error and then optimize the trajectory. The experiments are carried out to verify the proposed theory. The surface roughness and surface accuracy of the features were measured by Talysurf PGI 1240 and KEYENCE vhx900 respectively. The surface roughness and surface accuracy of the cylindrical sine wave groove micro feature surface are $0.1714 \mu \mathrm{m}$ and $1.32 \mu \mathrm{m}$ respectively. The surface roughness and surface accuracy of the cylindrical sinusoidal mesh micro feature surface are $0.1625 \mu \mathrm{m}$ and $1.8 \mu \mathrm{m}$ respectively. The results meet expectations and verify the reliability of the error theory and the trajectory optimization theory.
\end{abstract}

\section{Keywords:}

Cylindrical sine wave groove micro feature surface (CSWGS), Cylindrical sinusoidal mesh micro feature surface (CSMS), Single point diamond turning, Micro-feature surface modeling, Turning trajectory planning, Error theories

\section{Introduction}

It is of great significance to analyze and research micro features because it plays an important role in application to optics, biomimetics, medicine, communication and other fields. Recent advance in four reflective optical systems and optical lens always depend on the surface topographies and qualities of micro features to achieve better optical paths and benefits. In many other cases, the accuracy of microfeature surface needs to reach the accuracy of nanometer surface roughness and micron or even submicron form error, which has become a focus of research for experts and scholars in related fields all over the world.

Many researchers have done a lot of studies in the field of cylindrical microfeature processing and 
error analysis. Li R. B. et al. [1] analyzed the ultra-precision machining method of optical microstructure, and they designed a software that is suitable for the generation of turning trajectory for micro-lens and micro-groove machining. Gao W. et al. [2] proposed a method for adjusting the position of a tool in fasttool servo turning of the cylindrical outer circle. Lu H. et al. [3] studied that when the rear angle of the tool is less than the critical value of the characteristic, and they proposed a theoretical calculation model to calculate the wavelength and amplitude of the slot caused by the interference between the tool and the workpiece in the fast-tool servo turning of the micro-feature. Tauhiduzzaman M. et al. [4] studied the form error in diamond turning, and they thought the relative vibration between cutting tool and workpiece is the main cause of the error, and it is pointed out that the unbalanced spindle of machine tool has the most influence on machining of feature curved surface. Chen Y. L. et al. [5] studied a method of measuring micro-feature defects in fast tool servo lathe in real time, and the thrust force map was obtained by force sensor fluctuates abnormally and the defects were located, and then the micro-feature repair was carried out by more accurate tool path. Mak C. H. et al. [6] studied the effect of single point diamond turning on the machining accuracy of the V-shaped groove on the high-precision drum surface using different processing strategies, and they optimized the processing parameters including the turning depth and the turning times. Kong L. B. et al. [7] used single-point diamond turning with orthogonal slow-tool servo technology to process the corrugated micro-structure on the high-precision cylindrical surface under the 9 sets of different processing parameters. Mukaida M. et al. [8] used slow-tool servo diamond turning technology to process micro-lens array on single silicon material, and they studied the machining form error, surface morphology and turning force experimentally. Zhang X. et al. [9] studied the turning of cylindrical Fresnel lens as a die for pressing the Phoenix lens on the flexible matrix roll, and the cylindrical Fresnel lens was turned in both radial and axial direction of cylinders by four-axis linkage. Zhang G. Q. et al. [10] analyzed the centre error caused by the deviation between tool height and the spindle axis, and it could form a cone or cylinder boundary when the deviation appears. To determine and explore the tool interference they built a mathematical model and did some experiments, which have meaningful influence for controlling error. Wang Y. Q. et al. [11] proposed a scanning method for high-gradient free-form surface, which can generate mesh measuring path controlled by the planning deviation, and it has been verified by experiments. As can be seen from the above, there are many studies about how to improve surface quality during processing free surface on the plane, but few research about how to control the surface accuracy during processing free surface on the cylinder.

The remain part of this paper is arranged as: Modeling analysis is illustrated in Section 2. The tool parameter selection is described in Section 3. The error control theory of micro-feature processing is proposed in Section 4. The tool radius compensation is shown in Section 5. The experiments and corresponding results are presented in Section 6. Finally, conclusions are drawn in Section 7.

\section{Modeling of Cylindrical Microstructures}

There are many modeling methods for cylindrical microstructures, the two main methods are reverse modeling and forward modeling, this paper mainly studies forward modeling method. The Eq. 1 used to compound microfeatures by forward modeling is as follows: 


$$
\left\{\begin{array}{l}
g(x, y)=f\left(z_{1}\right) \\
h(x, y)=f\left(z_{2}\right) \\
G(x, y)=g(x, y)+h(x, y)
\end{array}\right.
$$

According to the forward modeling method of cylindrical microstructures, the theoretical model of cylindrical sine wave groove micro feature surface (CSWGS) and cylindrical sinusoidal mesh micro feature surface (CSMS) are carried out in this section. As shown in Table 1(a), the CSWGS is obtained by the combination of cylinder surface and sinusoidal line. The CSMS is obtained by the combination of CSWGS and radial sinusoidal feature surface as shown in Table 1(b). The expression of CSWGS is Eq. 2. The expression of radial sinusoidal feature surface is Eq. 3. The expression of CSMS is Eq. 4. The model diagram of the above micro-feature surfaces are shown in Table 1.

$$
\begin{gathered}
x_{1}=h \cdot \sin (\omega \cdot z)+R, \quad\left\{\begin{array}{l}
X_{(\mathrm{i}, \mathrm{j})}=x_{\mathrm{j}} \cdot \cos (2 \cdot \pi \cdot i / t) \\
Y_{(\mathrm{i}, \mathrm{j})}=x_{\mathrm{j}} \cdot \sin (2 \cdot \pi \cdot i / t) \\
Z_{(\mathrm{i}, \mathrm{j})}=z_{\mathrm{j}}
\end{array}\right. \\
x_{2}=R_{1} \cdot\left(h_{1}+h_{2} \cdot \sin \left(\omega_{1} \cdot t\right)\right) \\
\left\{\begin{array}{l}
x_{3 \mathrm{i}}=x_{1 \mathrm{i}} \cdot \cos \left(\theta_{\mathrm{i}}\right)+x_{2 \mathrm{i}} \cdot \cos \left(\theta_{\mathrm{i}}\right) \\
y_{3 \mathrm{i}}=x_{1 \mathrm{i}} \cdot \sin \left(\theta_{\mathrm{i}}\right)+x_{2 \mathrm{i}} \cdot \sin \left(\theta_{\mathrm{i}}\right) \\
z_{3 \mathrm{i}}=\Delta z \cdot i
\end{array}\right.
\end{gathered}
$$

Table 1. The model diagram and bus expression of micro-feature surfaces

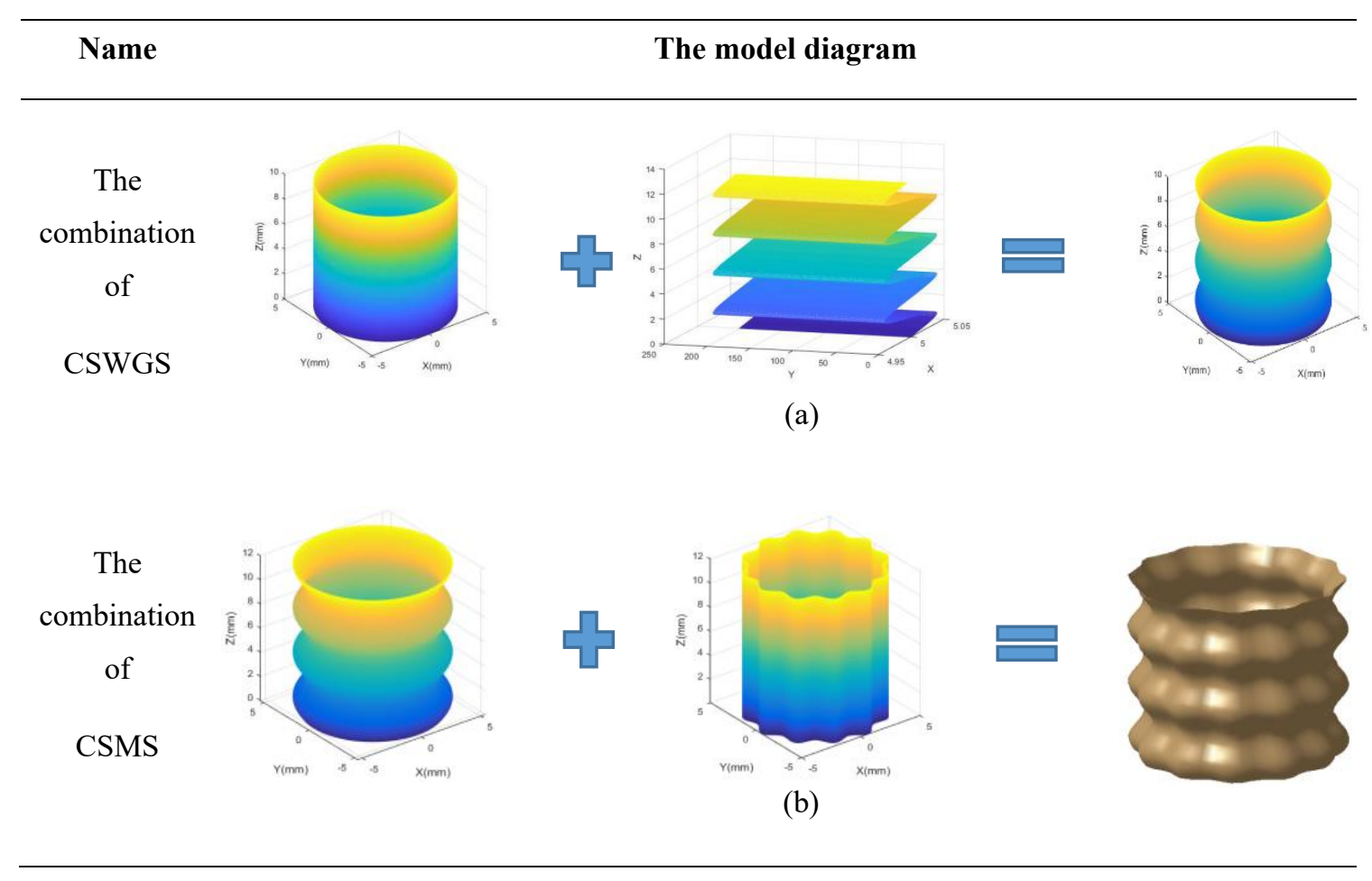

3. The Choice of Geometric Parameters of Diamond Tools 
In order to compensate the tool radius conveniently in the subsequent work, conical diamond tool is selected. The selection of correct tool parameters is very important for turning. The tool radius $r$, tool wrap angle $\alpha$, tool rake angle $\beta$ and tool rear angle $\gamma$ are analyzed in this section.

For machining in CSWGS and CSMS tool turning, the principle diagram of diamond turning is displayed in Fig. 1 and Fig. 3 respectively. Figure 2 and Figure 4 gives the expansion graph about the cutting procedure of CSWGS and CSMS respectively, the red track line is the tool contact turning trajectory, point $P_{\mathrm{i}}$ is the tool turning contact point.

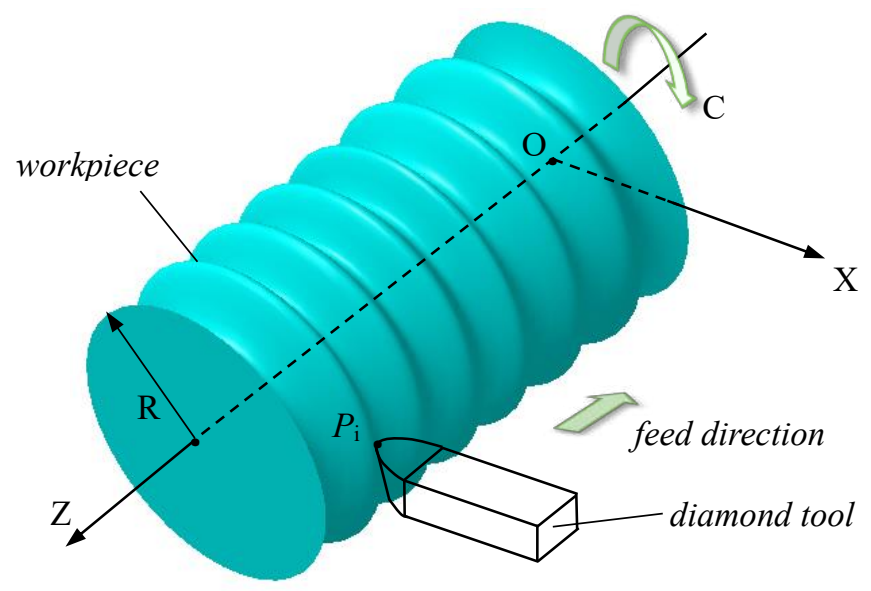

Fig. 1. The cutting principle diagram of CSWGS

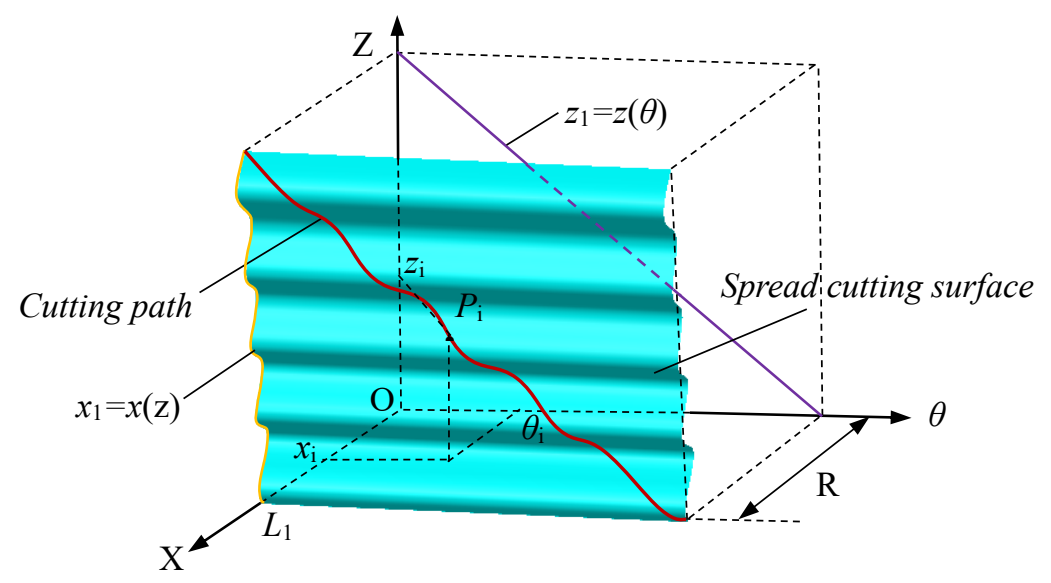

Fig. 2. The expanded view of cutting procedure of CSWGS 


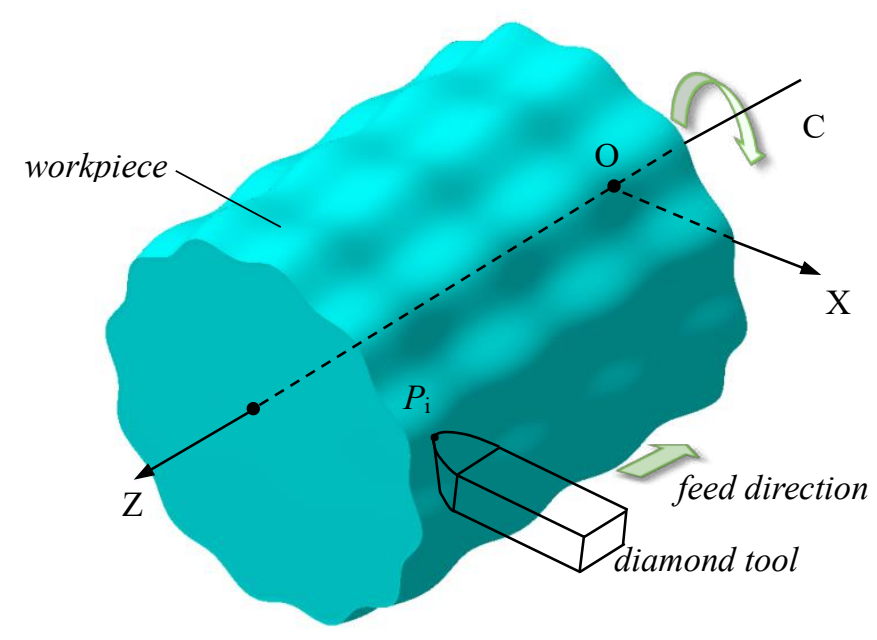

Fig. 3. The cutting principle diagram of CSMS

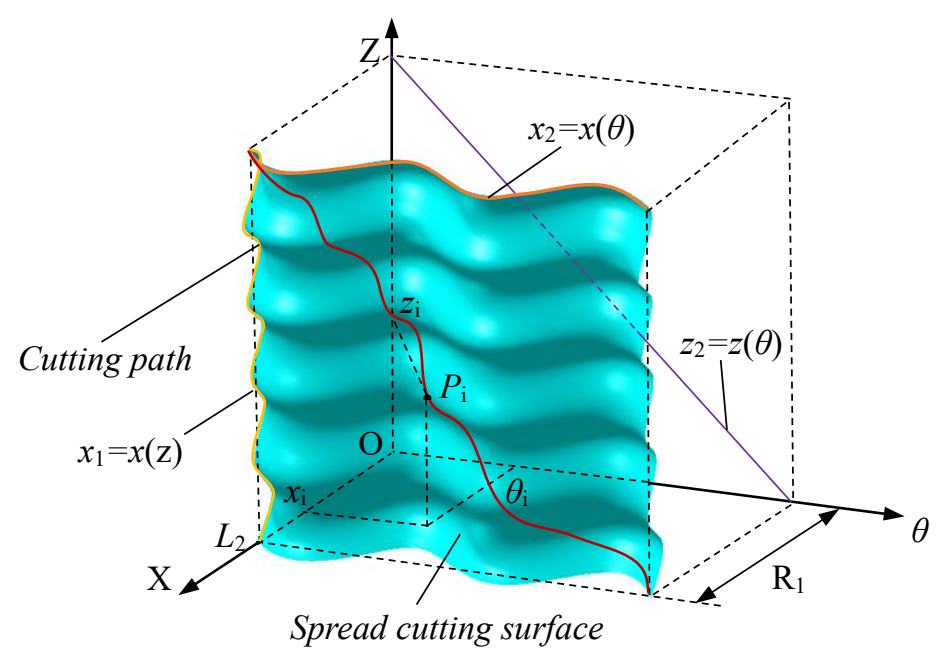

Fig. 4. The expanded view of cutting procedure of CSMS

In the turning process, the tool is fed along the workpiece axis, and the curvature at each point on the micro-feature axial bus needs to be calculated, and then the tool radius is selected. For CSWGS, only the curvature calculation shall be performed for the busbar. For CSMS, it is required to divide the characteristics within one cycle in the circumferential direction. Set the dividing angle as $\theta$, then the curve equation $\rho=f\left(z, \theta_{i}\right)$ fitting shall be carried out on the data of the micro-feature surface corresponding to $\theta_{0}, \theta_{1}, \theta_{2} \cdots \theta_{n}$, where, the radius of $\theta_{\mathrm{i}}$ is constant, $z$ is the variable, and then all turning points on each bus equation shall be calculated to calculate the radius of curvature and the cutter radius $r$.

Curvature of the curve is calculated as shown in Eq. 5. The tool wrap angle and tool rear angle can be calculated in the same way as the tool radius, whose computational method and mathematical analysis modeling are shown in Table 2.

Table 2. The mathematical analysis modeling and equation of diamond cutter parameters 


\begin{tabular}{lccc}
\hline Tool radius $r$ & Tool wrap angle $\alpha$ & Tool rear angle $\gamma$ \\
$K=\frac{\left|f^{\prime \prime \prime}\right|}{\left(1+f^{\prime 2}\right)^{3 / 2}}$ & $(5)$ & $\gamma_{i}=\left|\arctan \left(f^{\prime}\right)\right|$ \\
$r \leq \min \left\{\frac{1}{K_{0}}, \frac{1}{K_{1}}, \frac{1}{K_{2}} \cdots \frac{1}{K_{n}}\right\}$ & $(6) \quad\left\{\begin{array}{l}\alpha_{\mathrm{i}}=\left|\arctan \left(f^{\prime}\right)\right| \\
\alpha \geq 2 \max \left\{\alpha_{0}, \alpha_{1}, \alpha_{2} \cdots \alpha_{n}\right\}\end{array}\right.$ & $\gamma \geq \max \left\{\gamma_{0}, \gamma_{1}, \gamma_{2} \cdots \gamma_{n}\right\}(9)$
\end{tabular}

As shown in Fig. 5 (a) and Fig. 6 (a), the pitch $l j$ decides the tool wrap angle $\alpha$ and tool radius $r$, the direction of turning feed decides the tool rear angle $\gamma$ and tool rake angle $\beta$.

As shown in Fig. 5 (b), when the selected tool radius $r$ is greater than the curvature radius at a cutting point, the cutting interference occurs, and the larger the tool wrap angle $\alpha$ is, the longer the effective turning edge length of the tool tip is. On the $\gamma$ surface, when the tool radius is $r_{2}$, the tool wrap angle $\alpha$ is $\alpha_{1}$, there is a cutting interference at point $P_{\mathrm{i} 2}$. The wrap angle $\alpha_{2}$ at point $P_{\mathrm{i} 3}$ is greater than that $\alpha_{1}$ at point $P_{\mathrm{i} 1}$, and the effective turning edge length is longer.

As shown in Fig. 6 (b), the choice of tool rear angle $\gamma$ and tool rake angle $\beta$ may influence if the tool face will interfere with the machined surface. On the $\gamma^{\prime}$ surface, there is cutting interference at $P_{\mathrm{i} 1}$ and $P_{\mathrm{i} 4}$ points, and the tool rear angle $\gamma_{1}$ of point $P_{\mathrm{i} 1}$ is too small, and the tool rake angle $\beta_{2}$ of point $P_{\mathrm{i} 4}$ is too large. The rake angle at point $P_{\mathrm{i} 3}$ is 0 .

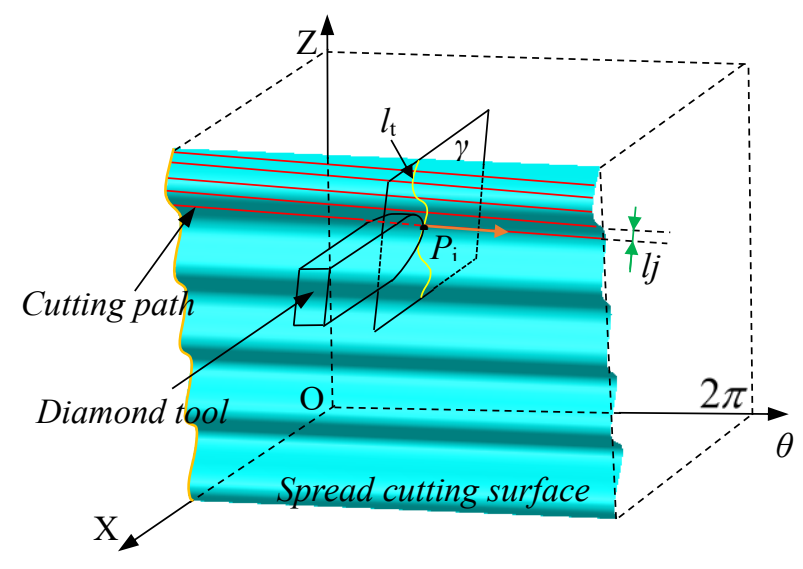

(a)

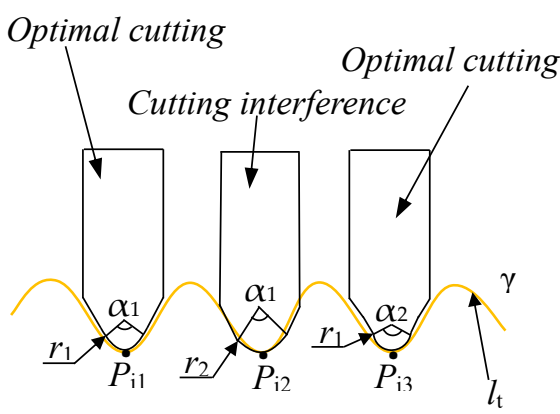

(b)

Fig. 5. Tool wrap angle $\alpha$ and tool radius $r$ of diamond tool 


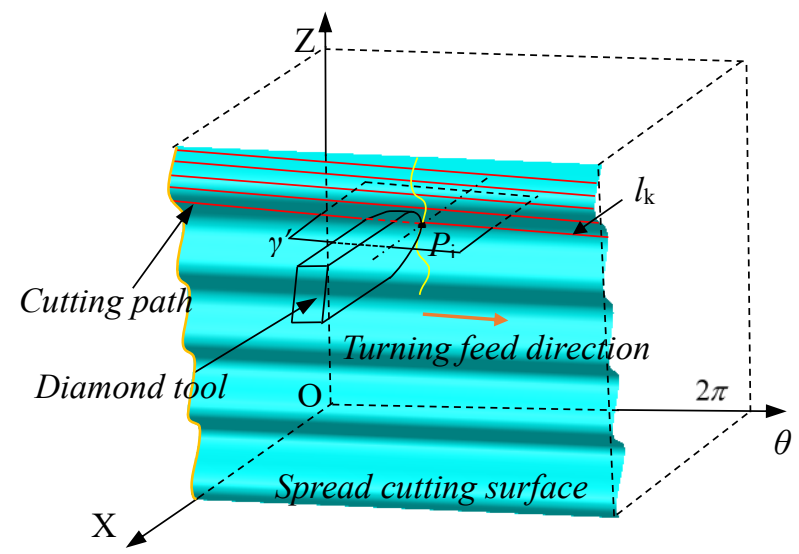

(a)

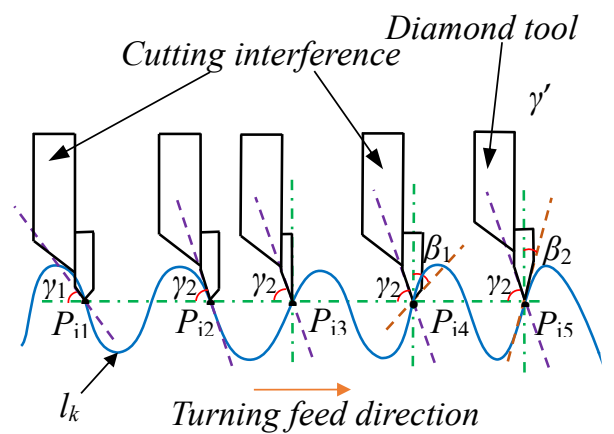

(b)

Fig. 6. Tool rear angle $\gamma$ and tool rake angle $\beta$ of diamond tool

\section{Error Control Theory of Micro-feature Processing}

The machining error caused by trajectory planning is the most important influence on machining accuracy in machining process. The establishment of error model are studied in this chapter, including residual height error and chord error.

\subsection{Establishment of Mathematical Model of residual height error}

Residual height error is caused by the circular arc at the tool tip of the diamond tool. The residual workpiece material will be produced between the adjacent tool contacts between the axial two adjacent turning tracks, resulting in the turning residual height error.

To calculate the residual height error, it is necessary to obtain the characteristic axial curve equation. For rotating body, it is the bus equation. For non-rotating body, curve equations need to be obtained by fitting the axial data of curved surfaces. Then the curvature radius $R_{\mathrm{QL}}$ and curvature at the corresponding point are calculated by axial curve equation, the positive and negative curvature is judged and the residual height error $R_{\text {er }}$ of three different cases is calculated by Eq. 10-12, when the curvature equals zero, the equation is 10 , when the curvature is less than zero (convex), the equation is 11 , when the curvature is greater than zero (concave), the equation is 12 . The theoretical analysis of the $R_{\text {er }}$ is shown in Fig. 7.

While $r$ is the arc radius of the tool tip of the selected tool, $f$ is the axial feed of the tool for each turning workpiece in the machining process, which is pitch. Based on the calculation of the above three cases, the feed $f$ can be reversed by Eq. 13, 14 .

$$
\begin{gathered}
R_{\mathrm{er}}=r-\sqrt{r^{2}-(f / 2)^{2}} \\
R_{\mathrm{er}}=\sqrt{\left(R_{\mathrm{QL}}+r\right)^{2}-(f / 2)^{2}}-\sqrt{r^{2}-(f / 2)^{2}}-R_{\mathrm{QL}} \\
R_{\mathrm{er}}=R_{\mathrm{QL}}-\sqrt{\left(R_{\mathrm{QL}}-r\right)^{2}-(f / 2)^{2}}-\sqrt{r^{2}-(f / 2)^{2}}
\end{gathered}
$$




$$
\left\{\begin{array}{l}
f_{1}=\sqrt{-R_{\mathrm{er}}\left(R_{\mathrm{er}}-2 R\right)\left(R_{\mathrm{er}}-2 r\right)\left(R_{\mathrm{er}}-2 R+2 r\right)} /\left(R_{\mathrm{er}}-R\right) \\
f_{2}=2 \sqrt{-R_{\mathrm{er}}\left(R_{\mathrm{er}}-2 r\right)} \\
f_{3}=\sqrt{-R_{\mathrm{er}}\left(R_{\mathrm{er}}+2 R\right)\left(R_{\mathrm{er}}-2 r\right)\left(R_{\mathrm{er}}+2 R+2 r\right)} /\left(R_{\mathrm{er}}+R\right)
\end{array}\right.
$$

$$
f=\min \left\{f_{1}, f_{2}, f_{3}\right\}
$$

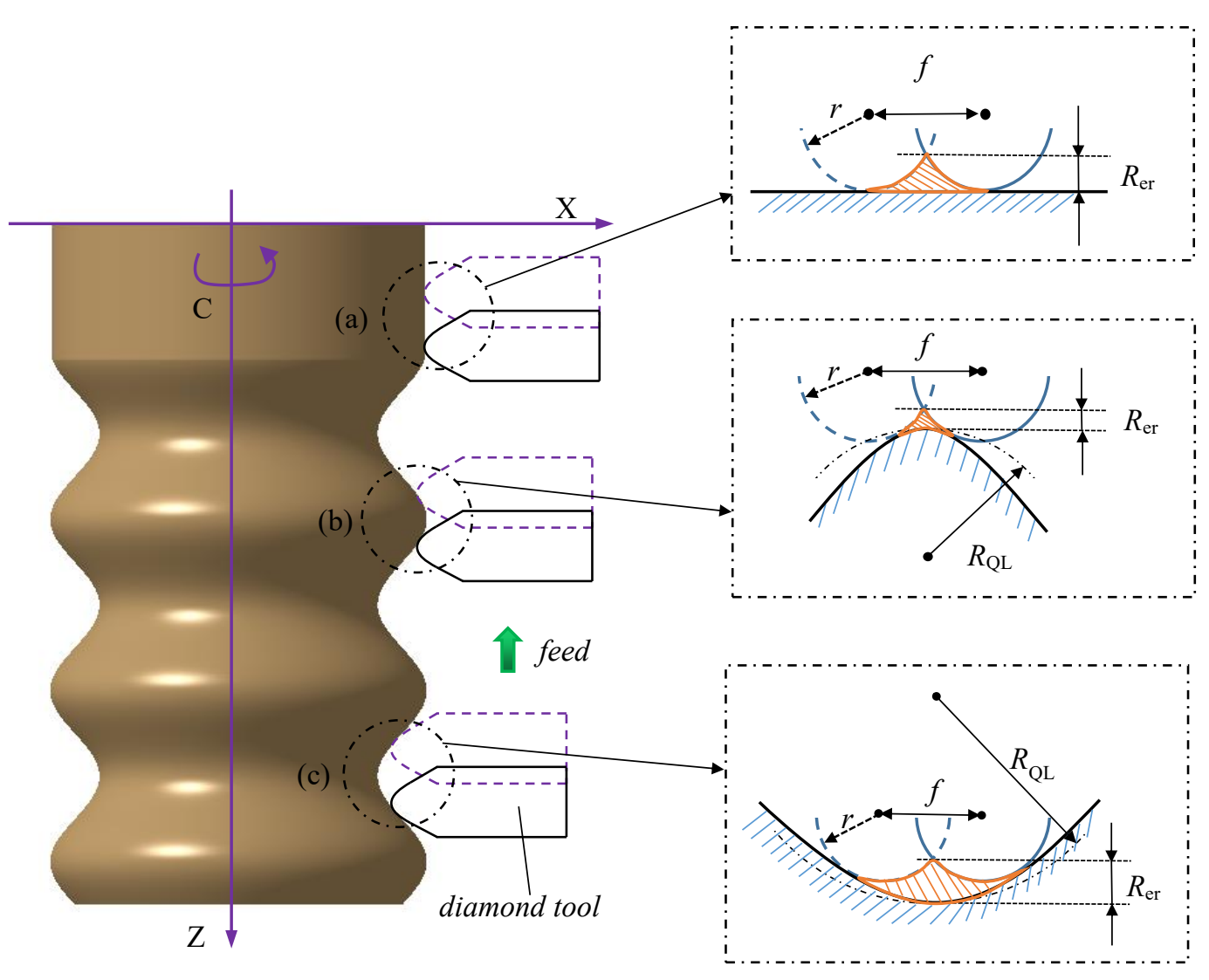

(a)The curvature equals zero

(b)The curvature is less than zero

(c)The curvature is greater than zero

Fig. 7. Residual error between axial two tool contacts

\subsection{Establishment of Mathematical Theoretical Model of chord error}

In order to improve the machining accuracy of cylindrical micro-feature surface, the mathematical theoretical model of chord error $C_{\text {er }}$ is established in this section. Fig. 8 (a) shows the formation position of the chord error, which is enlarged as shown. Fig. 8 (b) shows the mathematical model of chord error calculation, where $L_{\mathrm{i}}$ is the turning step, which is the distance between two adjacent turning contact points in the turning direction, $R_{\mathrm{i}}$ is the approximate radius of arc curvature at the calculated chord error.

According to Fig. 8 (b), the distance $L_{\mathrm{i}}$ shall be calculated by Eq. 15, of which $x, y, z$ is the spiral tool path tool contact. After solving the step size, the approximate arc curvature radius $R_{\mathrm{i}}$ needs to be calculated by Eq. 16 . 
Because the actual turning trajectory is composed of broken lines, and the turning trajectory is spirally fed on the cylindrical micro-feature surface, so there are three cases of chord error as shown in Fig. 8 (a).

$$
\begin{gathered}
L_{\mathrm{i}}=\sqrt{\left(x_{\mathrm{i}}-x_{\mathrm{i}+1}\right)^{2}+\left(y_{\mathrm{i}}-y_{\mathrm{i}+1}\right)^{2}+\left(z_{\mathrm{i}}-z_{\mathrm{i}+1}\right)^{2}} \\
R_{\mathrm{i}}=\frac{\left|F^{\prime}\right|^{3}}{\left|F^{\prime} \times F^{\prime \prime}\right|} \quad F^{\prime}=\left(x^{\prime}, y^{\prime}, z^{\prime}\right), F^{\prime \prime}=\left(x^{\prime \prime}, y^{\prime \prime}, z^{\prime \prime}\right)
\end{gathered}
$$

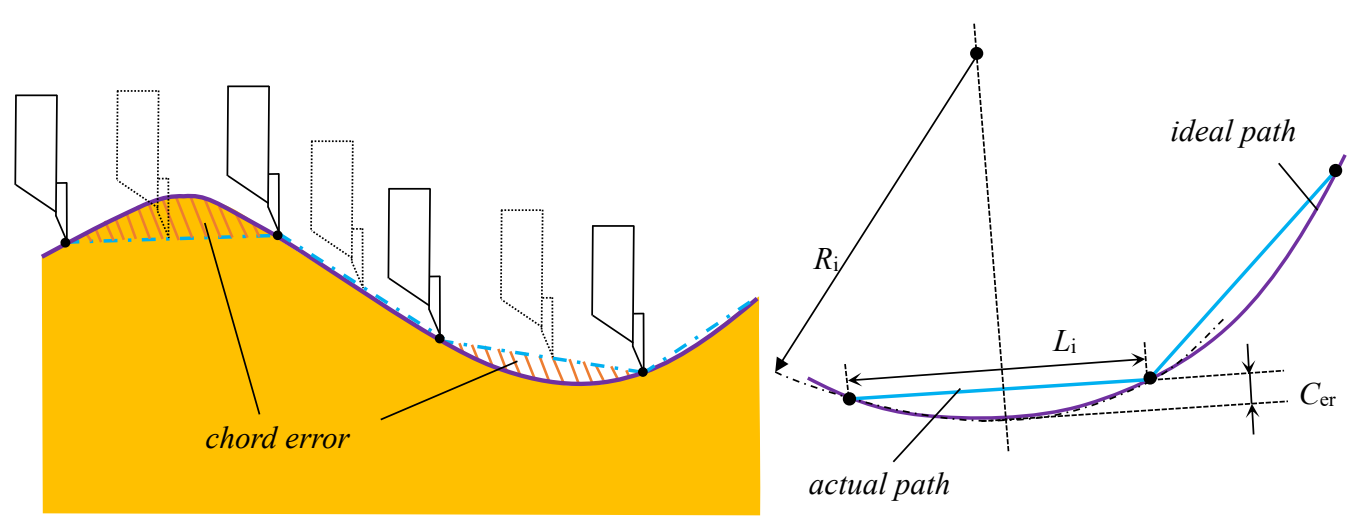

(a) chord error local magnification diagram

(b) geometric Model of chord error

Fig. 8. Schematic diagram of chord error

When the turning broken line is on the protruding surface, this situation may cause over-cutting phenomenon, when the turning broken line is on the sunken part of the surface, it may lead to insufficient turning, the chord error $C_{\mathrm{er}}$ can be calculated by Eq. 17. And then the turning step $L_{\mathrm{i}}$ can be solved by Eq. 18. Constrained turning step $L_{\mathrm{i}}$ size is essentially a constraint on the $t$ of radial fraction parameters.

$$
\begin{gathered}
C_{\mathrm{er}}=\left|R-\sqrt{R^{2}-(L / 2)^{2}}\right| \\
L_{\mathrm{i}} \leq \sqrt{8 R \cdot C_{\mathrm{er}}-4 C_{\mathrm{er}}{ }^{2}}
\end{gathered}
$$

\subsection{Trajectory Optimization}




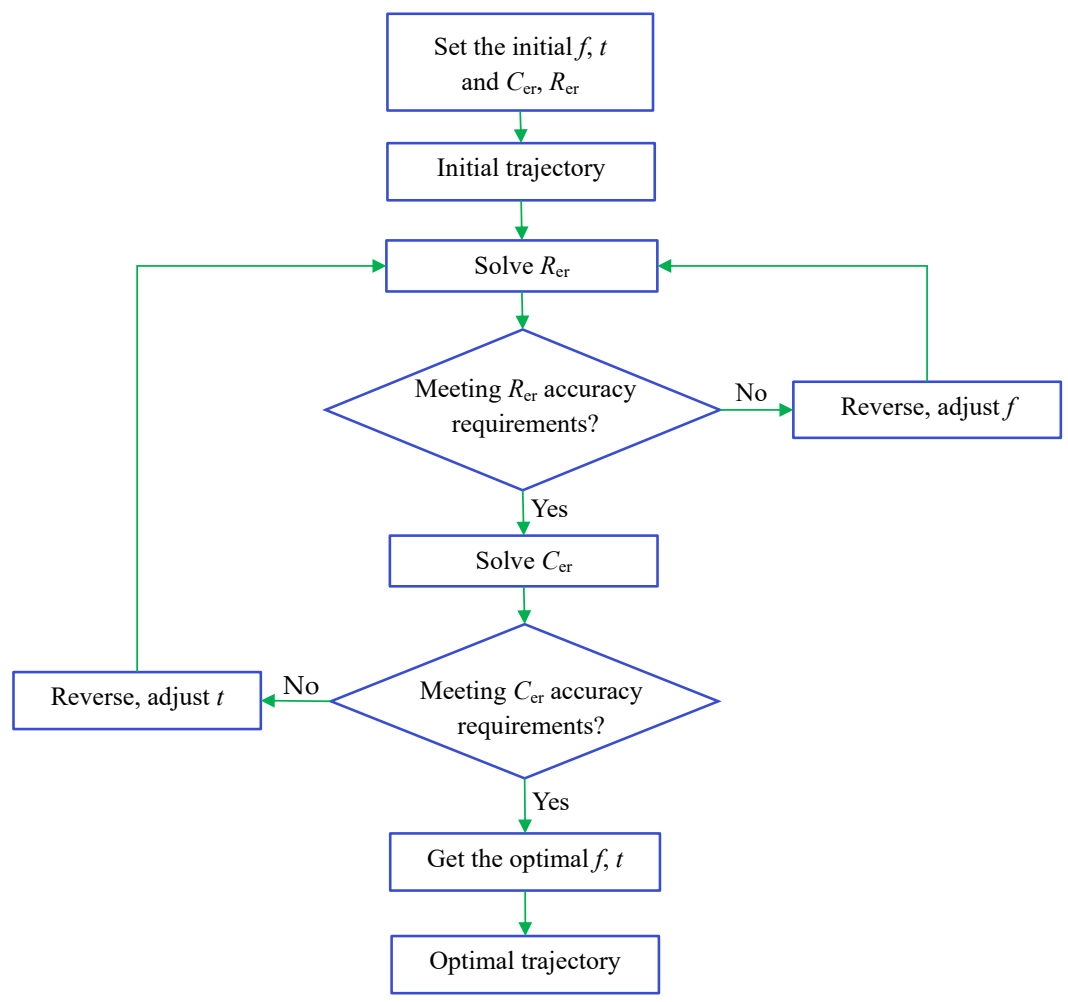

Fig. 9. Schematic diagram for optimal trajectory generation

The mathematical model of cylindrical micro-feature error has been established. The schematic diagram of generating the final optimal trajectory through error control is shown in Fig. 9. By controlling the error and adjusting the turning parameters repeatedly, the final turning trajectory is determined and the trajectory optimization is completed.

\section{Tool Radius Compensation}

The ultra-precision NC lathe needs to input the tool locus data of the turning trajectory, which is based on the tool contact path to compensate the tool radius in the normal vector direction of each point. The tool radius compensation is to compensate the tool contact points one by one in the normal vector direction of the tool contact points, which can get all the tool locus points. Tool radius compensation for rotating bodies such as CSWGS and non-rotating bodies such as CSMS is described in this section.

The angle $\theta$ is the angle between the normal vector and the $\mathrm{Z}$ axis at each point of the micro-feature surface. Let the micro-feature generatrix equation of the rotating body be $x=f(z)$, then the angle $\theta$ can be obtained by Eq. 19. By calculating the angle $\theta$, the tool radius compensation of the corresponding tool contact points can be carried out to obtain the tool locus points, which is shown in Eq. 20.

$$
\theta=\arctan \left(f^{\prime}(z)\right)
$$




$$
\left\{\begin{array}{l}
X_{\mathrm{i}}=R_{\mathrm{i}}+r \cdot \cos \left(\theta_{\mathrm{i}}\right) \cdot \cos (2 \cdot \pi \cdot i / t) \\
Y_{\mathrm{i}}=R_{\mathrm{i}}+r \cdot \cos \left(\theta_{\mathrm{i}}\right) \cdot \sin (2 \cdot \pi \cdot i / t) \\
Z_{\mathrm{i}}=z_{\mathrm{i}}-r \cdot \sin \left(\theta_{\mathrm{i}}\right)
\end{array}\right.
$$

While $R_{\mathrm{i}}$ is the distance from each tool contact point to the axis of the workpiece, the expression is $R_{\mathrm{i}}=\sqrt{\left(x_{i}\right)^{2}+\left(y_{i}\right)^{2}}$.

For cylindrical non-rotating micro-features, another tool radius compensation method is needed, the concrete steps are as follows. First, set the parametric equation of space curve Eq. 21, then the tangent direction vector $T$ will be Eq. 22. The first-order derivative and the second-order derivative of $k$ are multiplied to obtain a sub-normal direction vector $B$, which is shown in Eq. 23. At last, the main normal vector $N$ is equal to the cross product of $B$ and $T$ by Eq. 24 .

$$
\begin{gathered}
k=(a \cdot \cos \alpha, a \cdot \sin \alpha, b \cdot \alpha) \\
T=k^{\prime}=(-a \cdot \sin \alpha, a \cdot \cos \alpha, b) \\
B=k^{\prime} \times k^{\prime \prime}=\left(b^{*} \sin \alpha,-b^{*} \cos \alpha, a\right) \\
N=B \times T=(\cos \alpha, \sin \alpha, 0)
\end{gathered}
$$

According to Eq. 21-24, the main normal vector $N$ at each point on the turning trajectory of the nonrotating body cutter contact can be calculated, then the cutter radius compensation can be carried out to obtain the tool position trajectory.

Based on the calculation method of micro-feature and non-rotating micro-feature tool site of cylindrical rotary body described above, the calculation results are obtained as shown in Fig. 10, 11, and the effectiveness of the above method for solving the micro-feature tool locus points can be verified by turning the tool contacts and tool locus points shown in the figure.
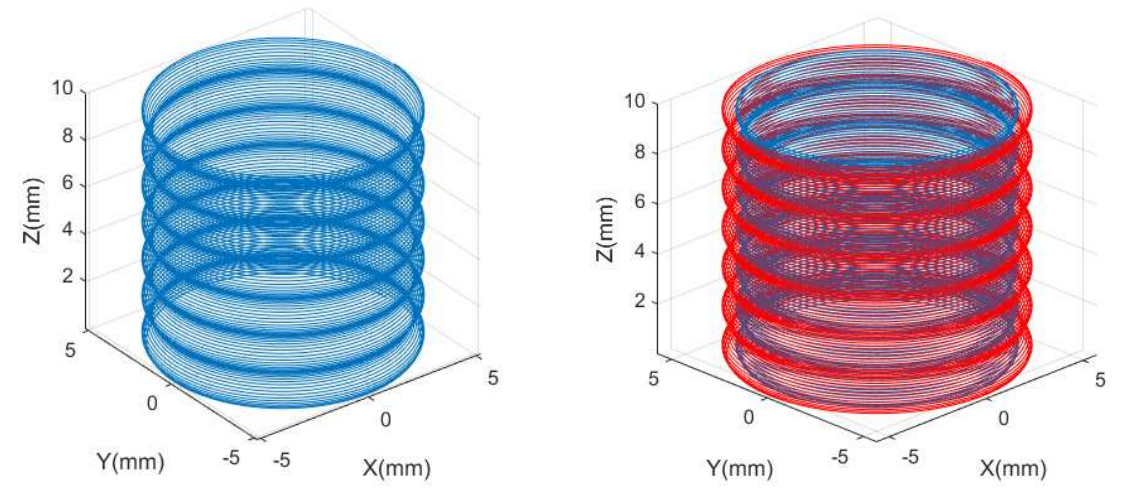

Fig. 10 . Blue tool contact trajectory (left) and red tool position trajectory (right) of CSWGS 

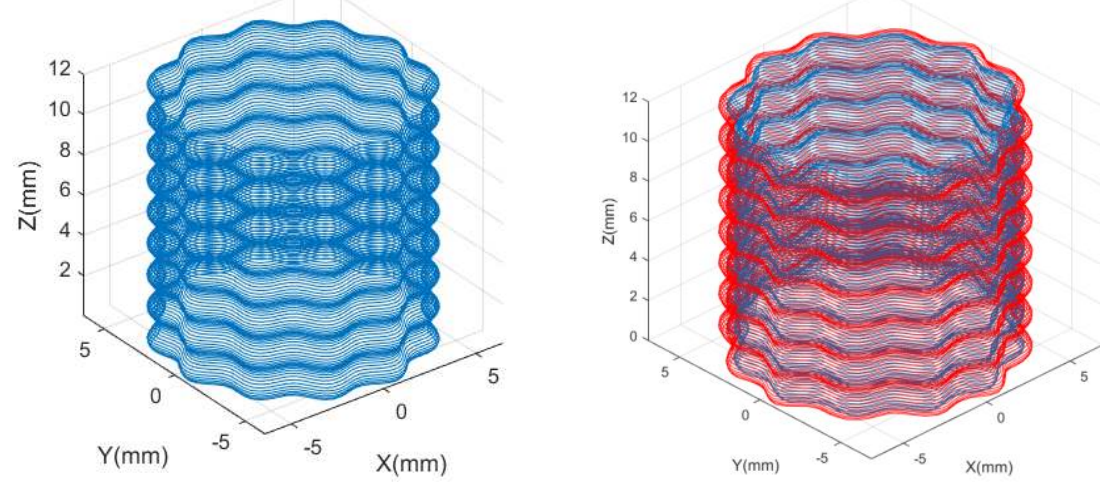

Fig. 11. Blue tool contact trajectory (left) and red tool position trajectory (right) of CSMS

\section{Experiments and Discussion}

On the basis of tool parameter optimization selection, turning trajectory planning, error model establishment and turning trajectory parameter optimization, two kinds of cylindrical micro-features are machined by using single point diamond slow cutter servo turning method. The two experiments are CSWGS experiment of rotary body and CSMS experiment of non-rotating body.

\subsection{Experimental Setup}

The machining error of cylindrical micro-feature (PV) is set to $1 \mu \mathrm{m}$, and the geometric parameters of turning tool are selected as shown in Table 3 . The type of precision turning machine is nanoform 250 , and the main parameters of the machine tool are shown in Table 4. The workpiece material is Al7075, and the diameter of the workpiece is $25 \mathrm{~mm}$, and the length $L$ of the workpiece is $6 \mathrm{~mm}$. The parameters of the two machined characteristic are shown in Table 5 and Table 6.

Table 3. Geometric parameters of diamond tools

\begin{tabular}{cccc}
\hline Tool parameter & Symbol & Parameter values & Units \\
\hline Tool rake angle & $\beta$ & 0 & $\circ$ \\
Tool rear angle & $\gamma$ & 1 & $\circ$ \\
Tool wrap angle & $\alpha$ & 120 & $\circ$ \\
Tool radius & $r$ & 0.506 & $\mathrm{~mm}$ \\
\hline
\end{tabular}

Table 4. Main parameters of nanoform250 Machine tool

\begin{tabular}{ccc}
\hline Main parameters of machine tool & Parameter values & Units \\
\hline C axis feedback resolution & 0.026 & arc seconds \\
C axis position accuracy & $+/-1$ & arc second \\
C axis maximum speed & 3000 & RPM
\end{tabular}


$\mathrm{X}, \mathrm{Z}$ axis stroke

$\mathrm{X}, \mathrm{Z}$ axis straightness

Position feedback Resolution of Linear

hydrostatic guideway
220

$\mathrm{mm}$

horizontal full stroke 0.2

$\mu \mathrm{m}$

$\mathrm{nm}$

0.016

Table 5. Required parameters of CSWGS

\begin{tabular}{cccc}
\hline Parameter name & Symbol & Parameter values & Units \\
\hline Sine wave slot amplitude & $h$ & 0.04 & $\mathrm{~mm}$ \\
Sinusoidal wave & $\omega$ & 4 & $\mathrm{rad} / \mathrm{s}$ \\
Characteristic radius & $R$ & 12.5 & $\mathrm{~mm}$ \\
Characteristic length & $L$ & 6 & $\mathrm{~mm}$ \\
Cutting points number & $t$ & 249 & Pts/Round \\
Pitch & $f$ & 0.0635 & $\mathrm{~mm}$ \\
\hline
\end{tabular}

Table 6. Required parameters of CSMS

\begin{tabular}{cccc}
\hline Parameter name & Symbol & Parameter values & Units \\
\hline Axial amplitude & $h$ & 0.02 & $\mathrm{~mm}$ \\
Axial period & $\omega$ & 4 & $\mathrm{rad} / \mathrm{s}$ \\
Axial feature & $R$ & 12.2 & $\mathrm{~mm}$ \\
First radial amplitude & $h_{1}$ & 0.03 & $\mathrm{~mm}$ \\
Second radial amplitude & $h_{2}$ & 0.02 & $\mathrm{~mm}$ \\
Radial period & $\omega_{1}$ & 12 & $\mathrm{rad} / \mathrm{s}$ \\
Radius & $R_{1}$ & 2.5 & $\mathrm{~mm}$ \\
Cutting points number & $t$ & 249 & $\mathrm{Pts} / \mathrm{Round}$ \\
Pitch & $f$ & 0.0635 & $\mathrm{~mm}$ \\
\hline
\end{tabular}

The workpiece is rough machined to get the cylindrical surface, and then refined on nanoform 250 ultra-precision machine tool. Before machining, the machine tool is aligned and the main shaft dynamic balance is adjusted to ensure the machining accuracy. The tool is equipped with LVDT optical tool alignment device to the tool process. After completing the two operations above, the machining trajectory data optimized by the error model are used to transform the Cartesian coordinates into cylindrical coordinates and input them into ultra-precision NC machine tools for micro-feature machining.

\subsection{Results and Discussions}


The surface accuracy of the two machined microfeatures is measured by Talysurf PGI 1240 instrument, which is shown in Fig. 12, 13. After processing the measured data, the measured data are matched with the theoretical modeling data, and the error between the two data is analyzed, which is regarded as the PV values of the machining characteristics.

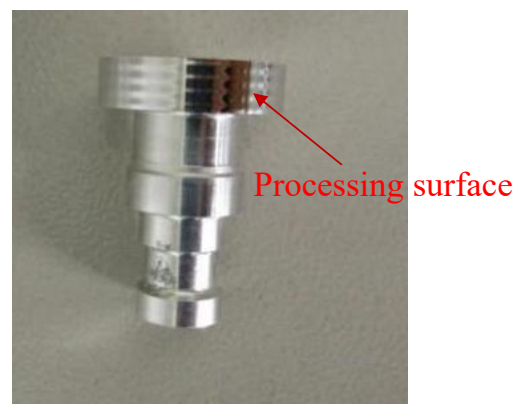

(a)

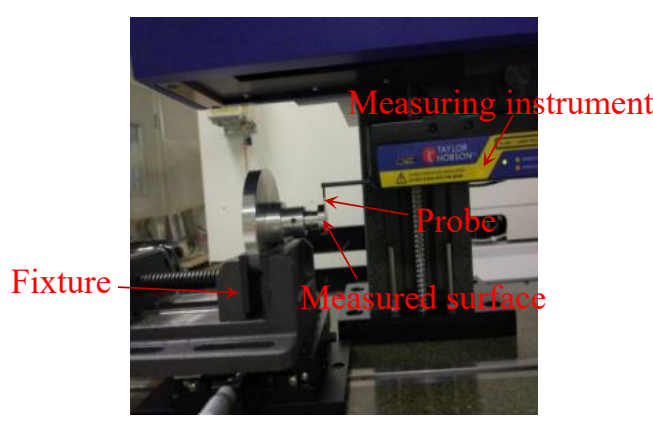

(b)

Fig. 12. Final processing object and microfeature measurement of CSWGS. (a) Final object; (b) Measurement.

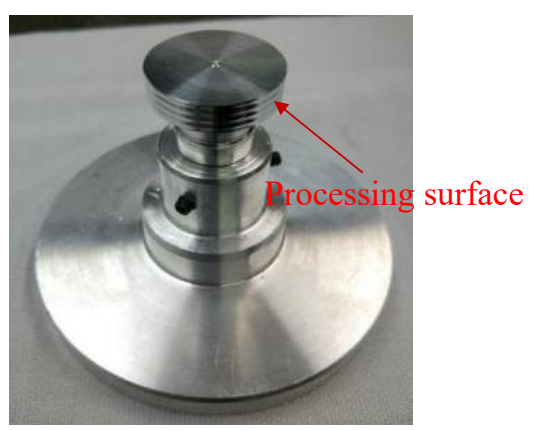

(a)

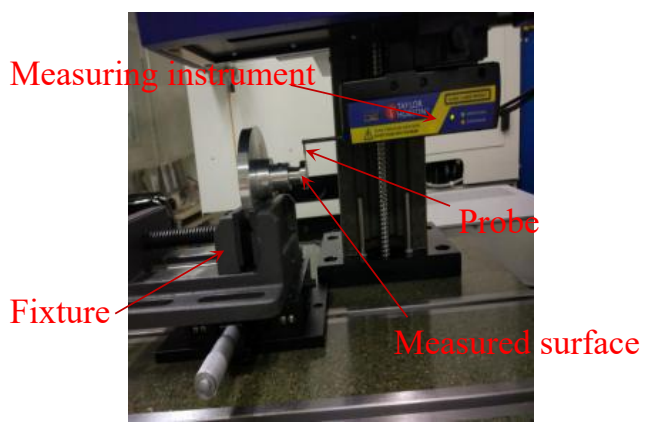

(b)

Fig. 13. Final processing object and microfeature measurement of CSMS (a) Final object; (b) Measurement.

The roughness measurement of CSWGS and CSMS are shown in Fig. 14 (a) and (b), and the measured roughness are $0.1714 \mu \mathrm{m}$ and $0.1625 \mu \mathrm{m}$ respectively. The surface morphology of CSWGS and CSMS were measured by Keenz vhx900 instrument, and the measured results are shown in Fig. 14 (c) and (d) respectively. The measured data and the theoretical values of CSWGS are shown in Fig. 14 (e). The radial error band of CSMS is shown in Fig. 14 (f). The deviation between measured data and theoretical data of CSWGS and CSMS are shown in Fig. 14 (g) and (h), and the PV values of them are $1.32 \mu \mathrm{m}$ and $1.8 \mu \mathrm{m}$ respectively, which have little difference with the setting error.
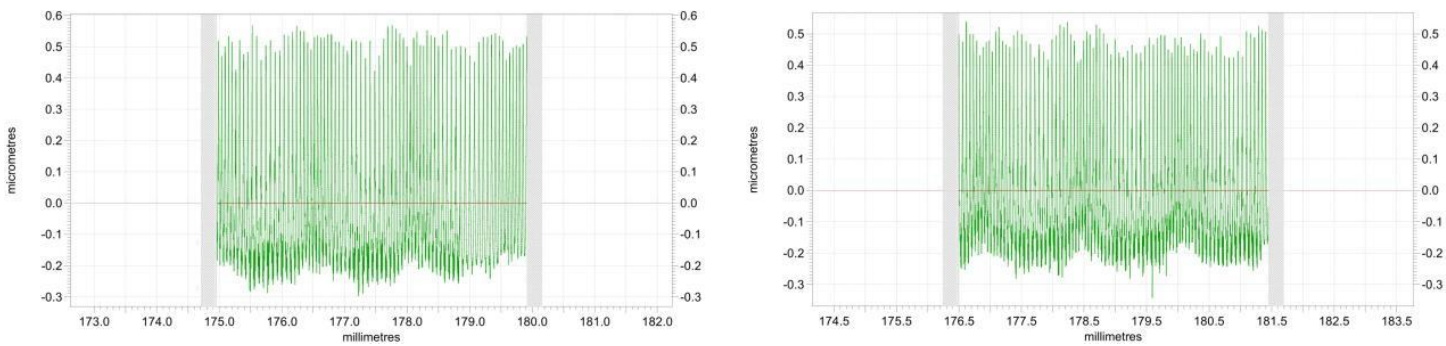


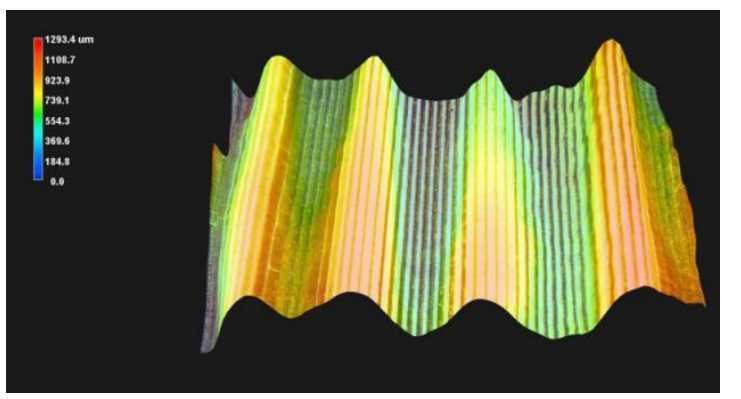

(c)

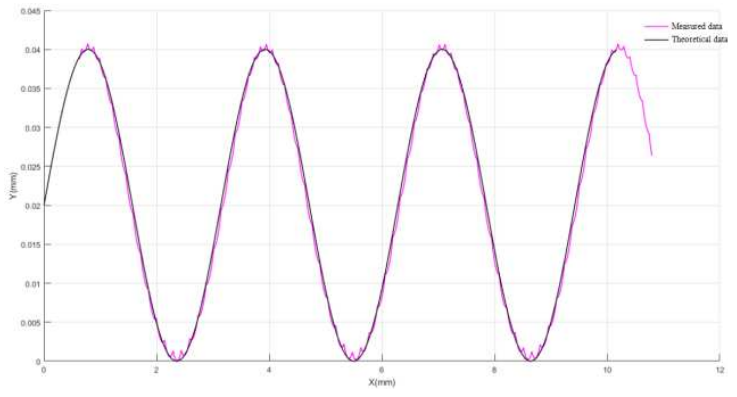

(e)

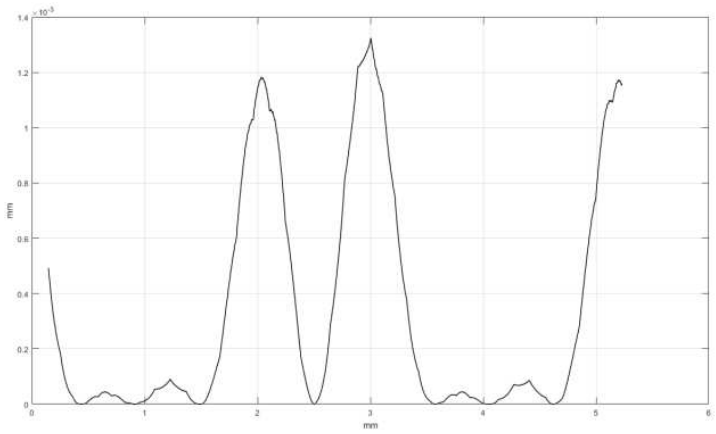

(g)

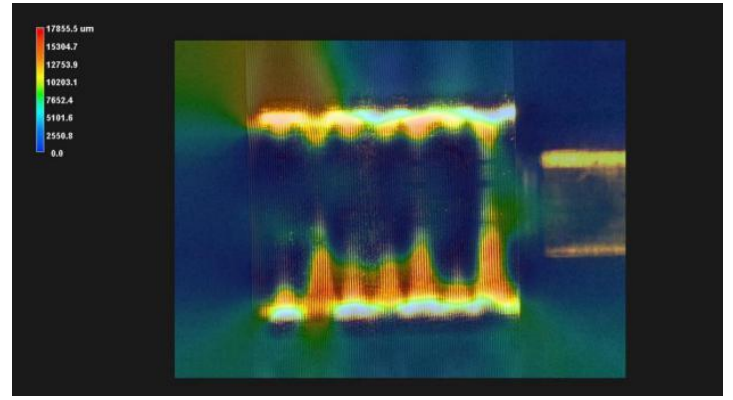

(d)

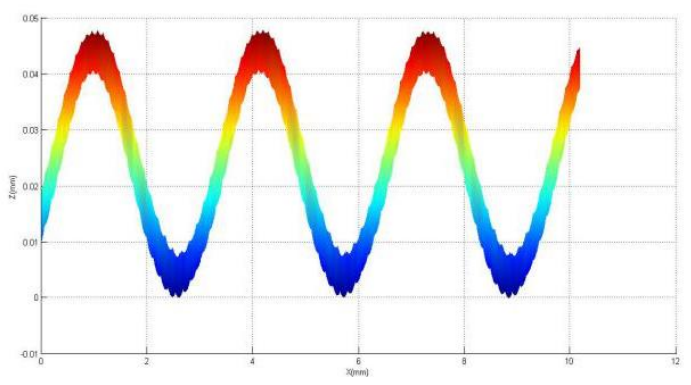

(f)

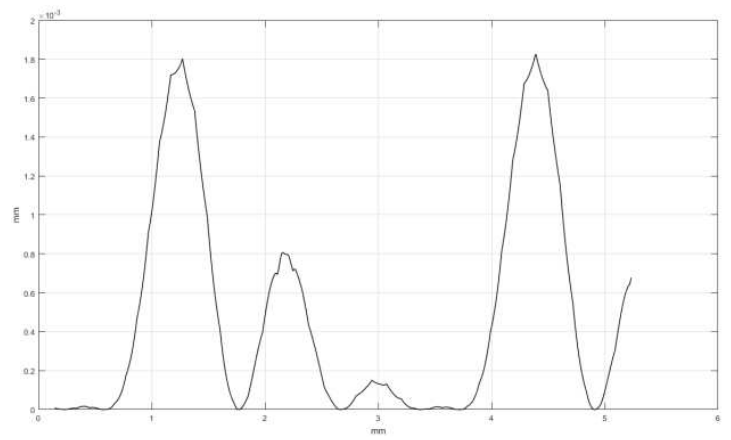

(h)

Fig. 14. Measurement and calculation results of CSWGS and CSMS

Experimental results show that all of them have high accuracy, which verifies the theory of trajectory planning, the method of optimizing turning trajectory parameters by error model and the reliability of micro-features of turning cylindrical and non-rotating body.

\section{Conclusions}


In this paper, the theory and technology of single point diamond turning with micro-features of cylindrical surface are studied. The research contents mainly include the optimization selection of geometric parameters of diamond tools, the modeling of cylindrical micro-feature surfaces and the generation principle of tool turning trajectory, the principle of tool radius compensation, and the optimization of machining trajectory parameters by establishing the theoretical model of turning error. The turning experiments of cylindrical sinusoidal groove and non-rotating cylindrical sinusoidal grid of rotating body are carried out by using nanoform 250 ultra-precision machine tool. Talysurf PGI 1240 instrument is used to measure the precision of workpiece surface, and then the obtained data are processed and analyzed. The machined feature surface morphology is measured by Keenz vhx900. The micro characteristic surface roughness (Ra) of CSWGS is $0.1714 \mu \mathrm{m}$, and the surface shape accuracy is $1.32 \mu \mathrm{m}$. The surface roughness of CSMS is $0.1625 \mu \mathrm{m}$, and the surface shape accuracy is $1.8 \mu \mathrm{m}$. All of them have high machining accuracy, which verifies the correctness of the theory proposed in this paper.

Authors Contributions Jingjin Li contributed to the process and summary of the study and wrote the manuscript. Shijun Ji and Ji Zhao made great guidance on the concept, direction and experiment of the research. Jingjin Li and Jianfeng Li designed and performed the experiment. Jingjin Li and Handa Dai tested the results and analyzed the data.

Funding This research work is supported by National Natural Science Foundation of China (Grant No 51775237), Key R\&D Projects of the Ministry of Science and Technology of China (Grant Nos. 2018YFB1107600 and 2017YFA0701200), Key Projects of Jilin Province Science and Technology Development Plan(Grant No. 20200401121GX) and Key scientific research project of Jilin Provincial Department of Education(Grant No. JJKH20200972KJ) of China.

Data availability The datasets used or analysed during the current study are available from the corresponding author on reasonable request.

Code availability Not applicable.

\section{Declarations}

Ethics approval and consent to participate Compliance with ethical standards. All authors agreed with the consent to participate. 
Consent to publish All authors have read and agreed to the published version of the manuscript.

Competing interests The authors declare no competing interest.

\section{References}

[1] Rongbin Li, Xue Du , Zhihui Zhang, etc. Ultra-precision machining technology of optical microstructure [D]. Nanotechnology and Precision Engineering, 2003.

[2] Gao W, Chen Y L, Lee K W, et al. Precision tool setting for fabrication of a microstructure array[J]. CIRP Annals, 2013, 62(1): 523-526.

[3] Lu H, Lee D, Kim J, et al. Modeling and machining evaluation of microstructure fabrication by fast tool servo-based diamond machining[J]. Precision engineering, 2014, 38(1): 212-216.

[4] Tauhiduzzaman M, Yip A, Veldhuis S C. Form error in diamond turning[J]. Precision Engineering, 2015, 42: 22-36.

[5] Chen Y L, Wang S, Shimizu Y, et al. An in-process measurement method for repair of defective microstructures by using a fast tool servo with a force sensor[J]. Precision Engineering, 2015, 39: $134-142$.

[6] Mak C H, Cheung C F, Ren M J, et al. A Study of Cutting Strategy in Single-Point Diamond Turning of Micro V-groove patterns on Precision Roller Drums[C]/Key Engineering Materials. Trans Tech Publications, 2015, 625: 742-747.

[7] Kong L B, Cheung C F, Lee W B. A theoretical and experimental investigation of orthogonal slow tool servo machining of wavy microstructured patterns on precision rollers[J]. Precision Engineering, 2016, 43: 315-327.

[8] Mukaida M, Yan J. Fabrication of hexagonal microlens arrays on single-crystal silicon using the tool-servo driven segment turning method[J]. Micromachines, 2017, 8(11): 323.

[9] Zhang X, Huang R, Liu K, et al. Rotating-tool diamond turning of Fresnel lenses on a roller mold for manufacturing of functional optical film[J]. Precision Engineering, 2018, 51: 445-457.

[10] Zhang G. Q., Dai Y. Q., Suet To, et al. Tool interference at workpiece centre in single-point diamond turning[J]. International Journal of Mechanical Sciences, 2019, 151: 1-12.

[11] Wang Y. Q., Xi M. M., Liu H. B., et al. On-machine noncontact scanning of high-gradient freeform surface using chromatic confocal probe on diamond turning machine[J]. Optics \& Laser Technology, 
2021, 134. 
Figures

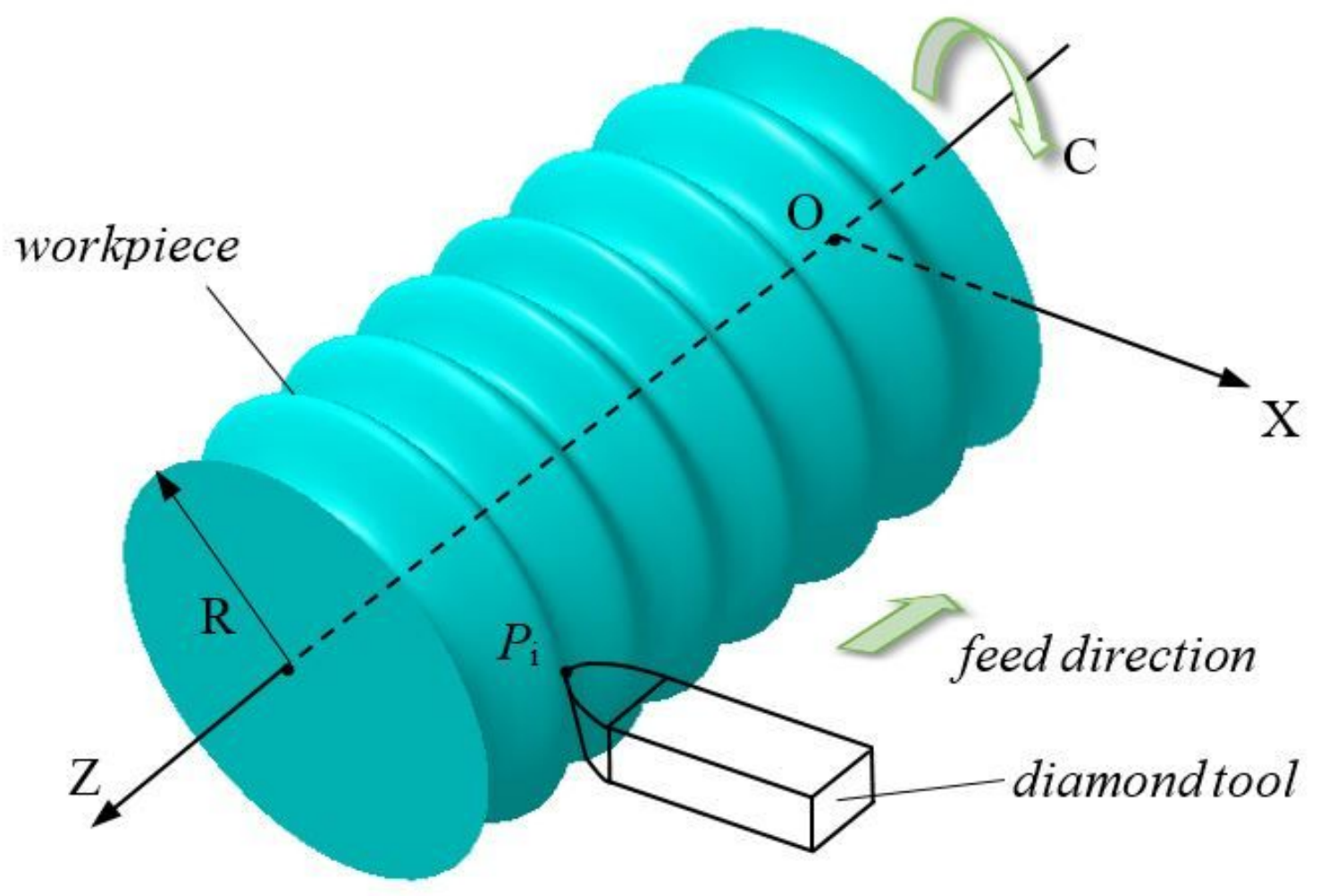

Figure 1

The cutting principle diagram of CSWGS 


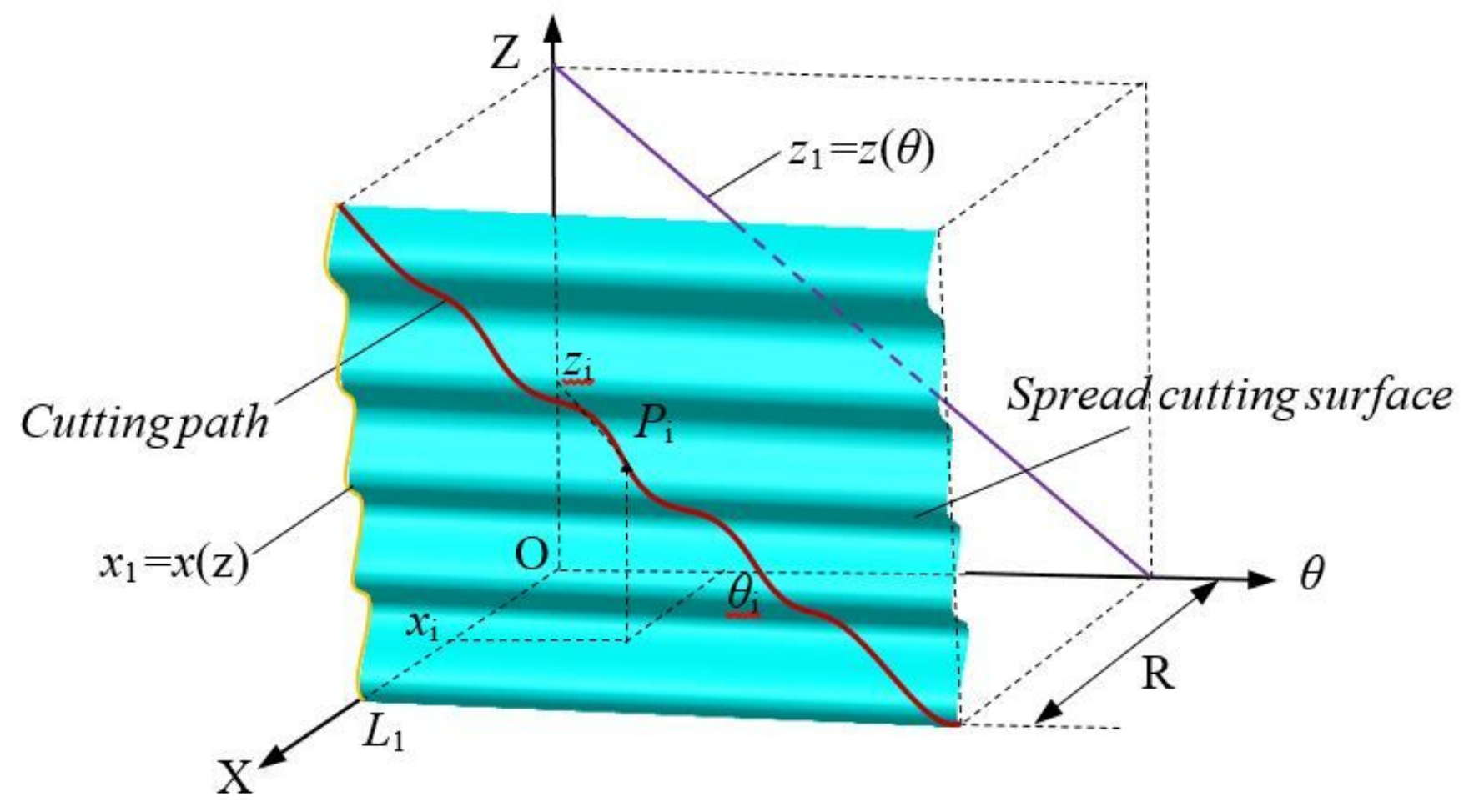

Figure 2

The expanded view of cutting procedure of CSWGS 


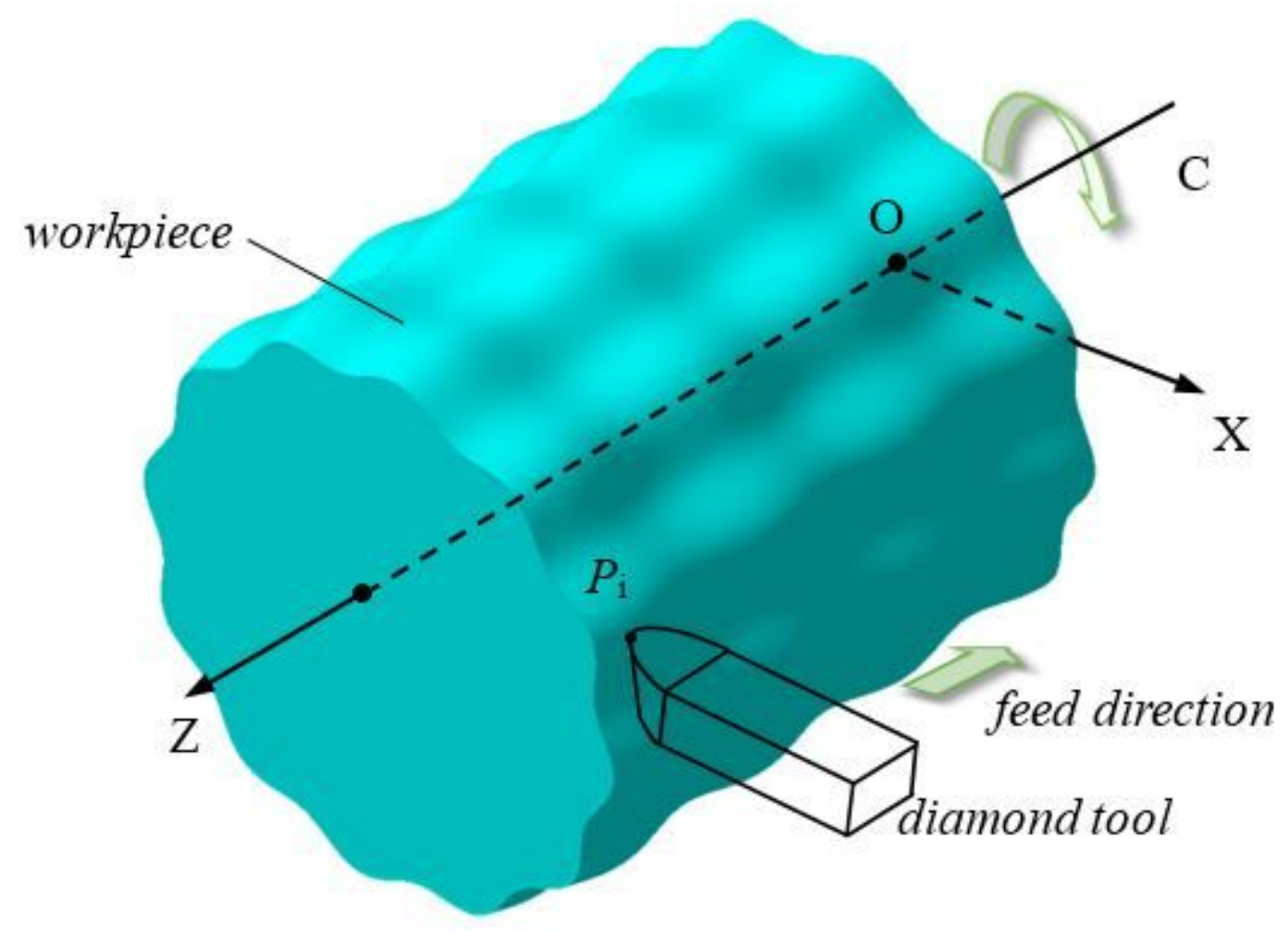

Figure 3

The cutting principle diagram of CSMS 


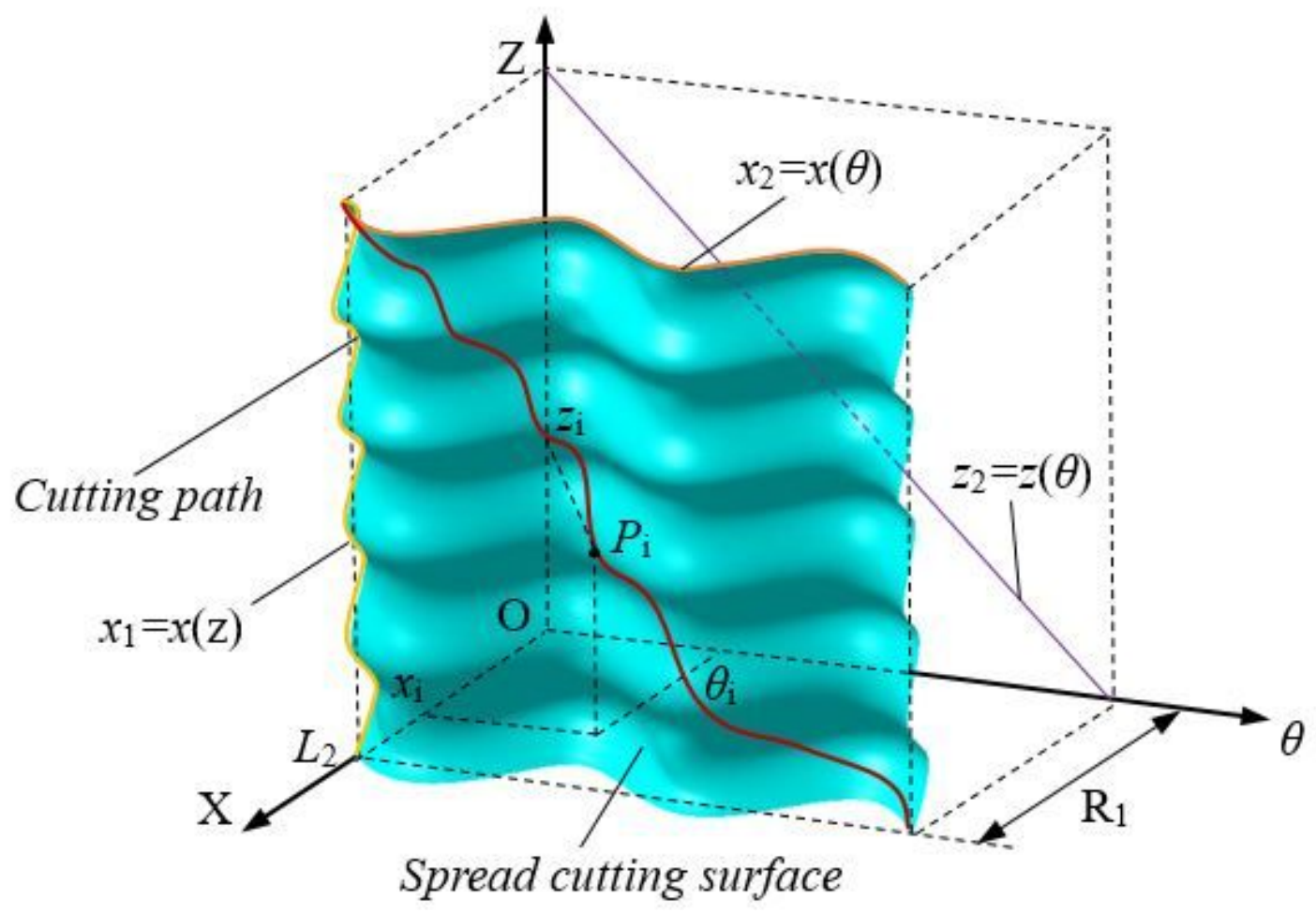

Figure 4

The expanded view of cutting procedure of CSMS

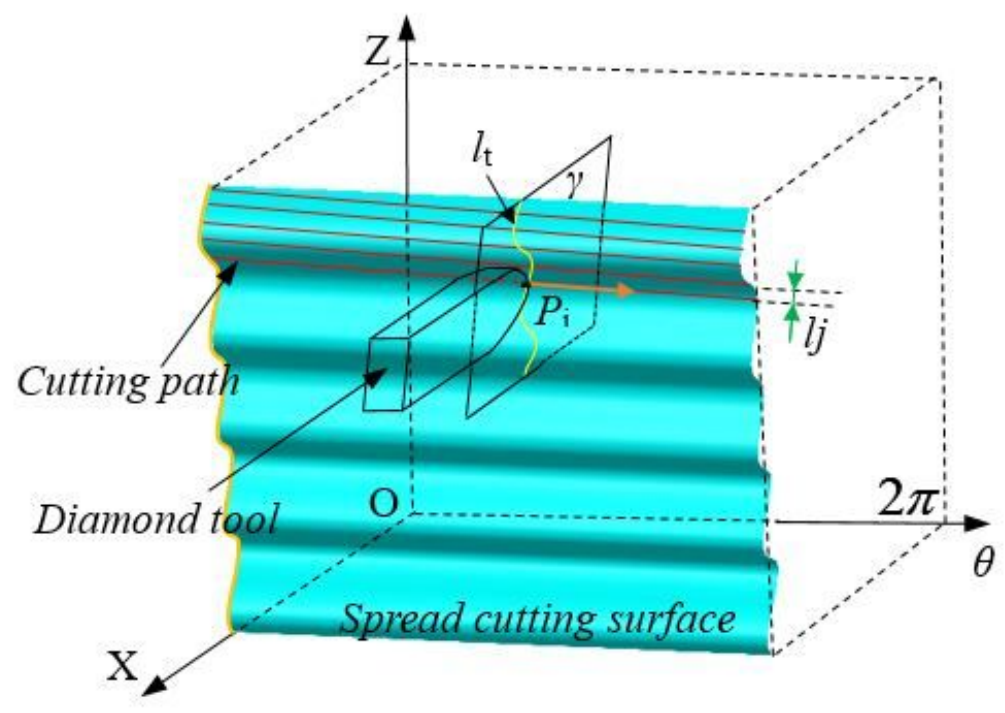

(a)

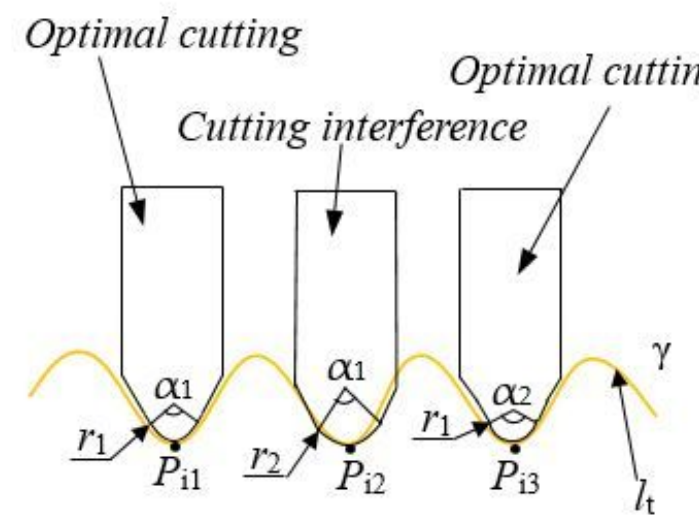

(b)

\section{Figure 5}

Tool wrap angle $\mathrm{a}$ and tool radius $\mathrm{r}$ of diamond tool 


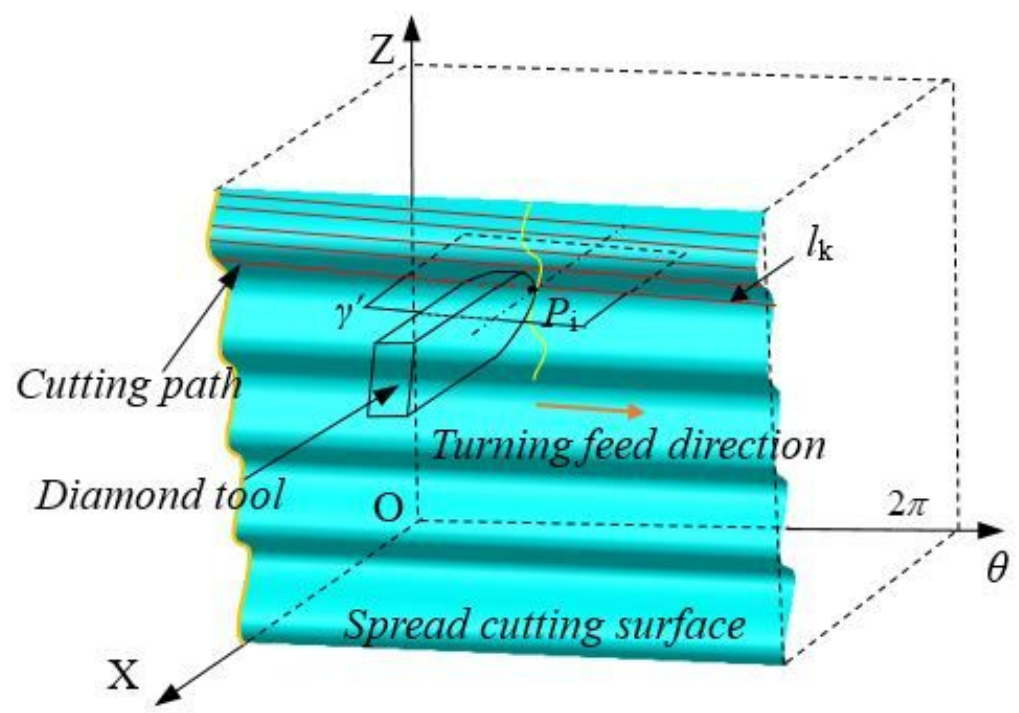

(a)

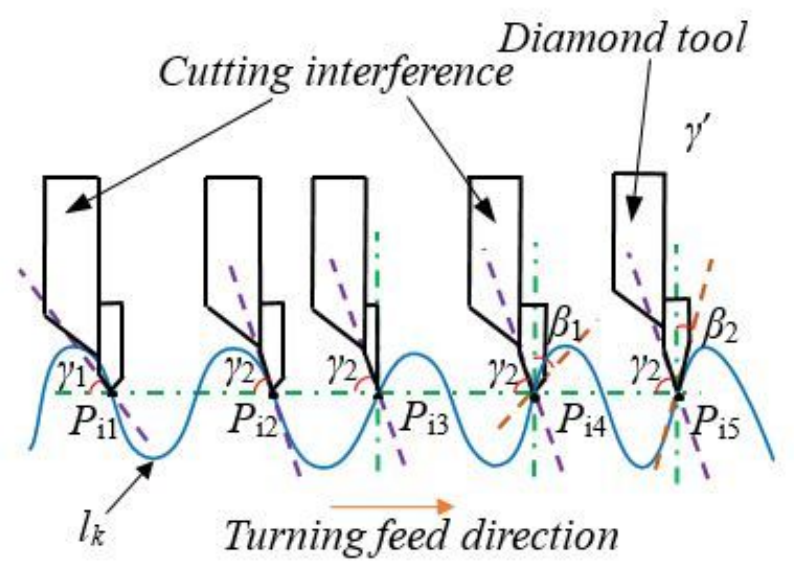

(b)

Figure 6

Tool rear angle $y$ and tool rake angle $\beta$ of diamond tool 


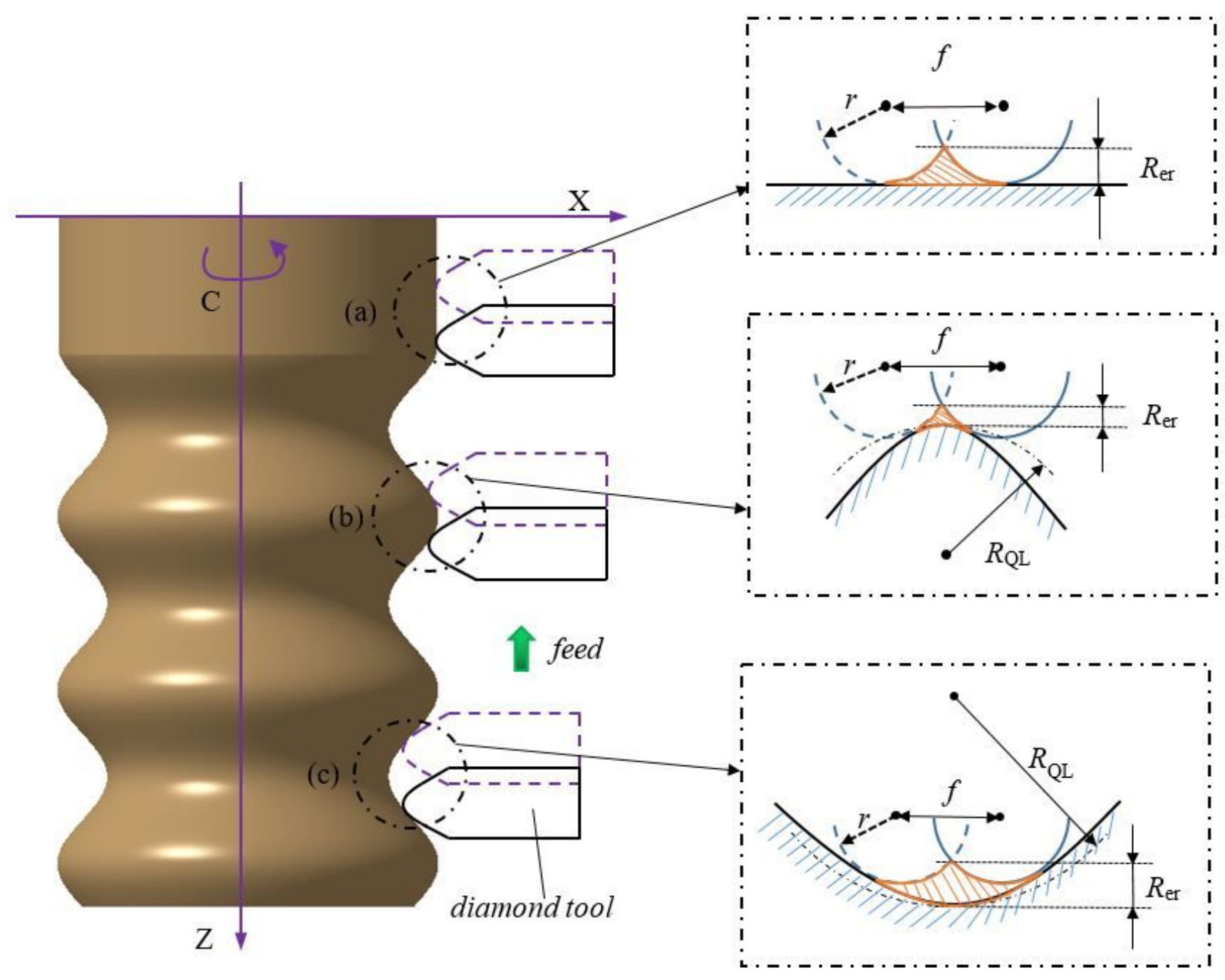

$\begin{array}{llll}\text { (a)The curvature equals zero } & \text { (b)The curvature is less than zero } & \text { (c)The curvature is greater than zero }\end{array}$

Figure 7

Residual error between axial two tool contacts 


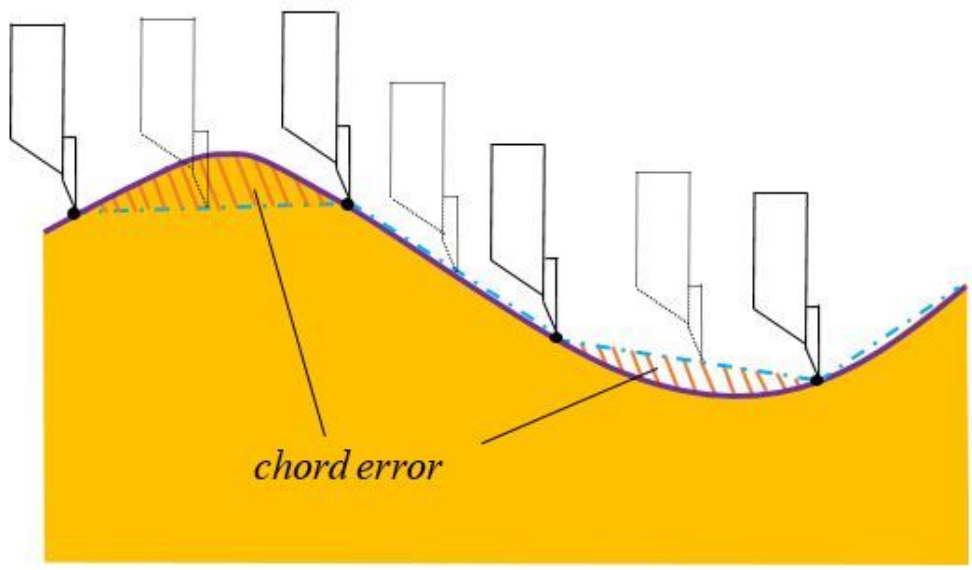

(a) chord error local magnification diagram

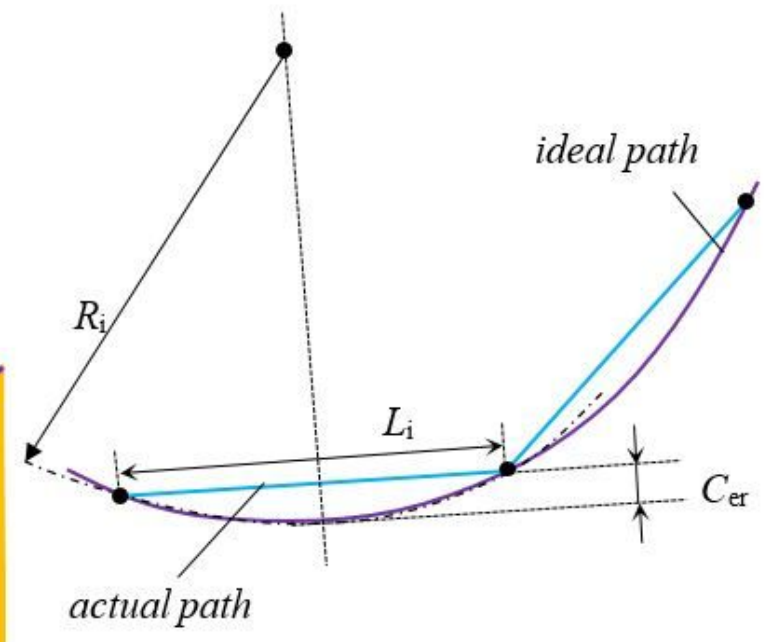

(b) geometric Model of chord error

\section{Figure 8}

Schematic diagram of chord error 


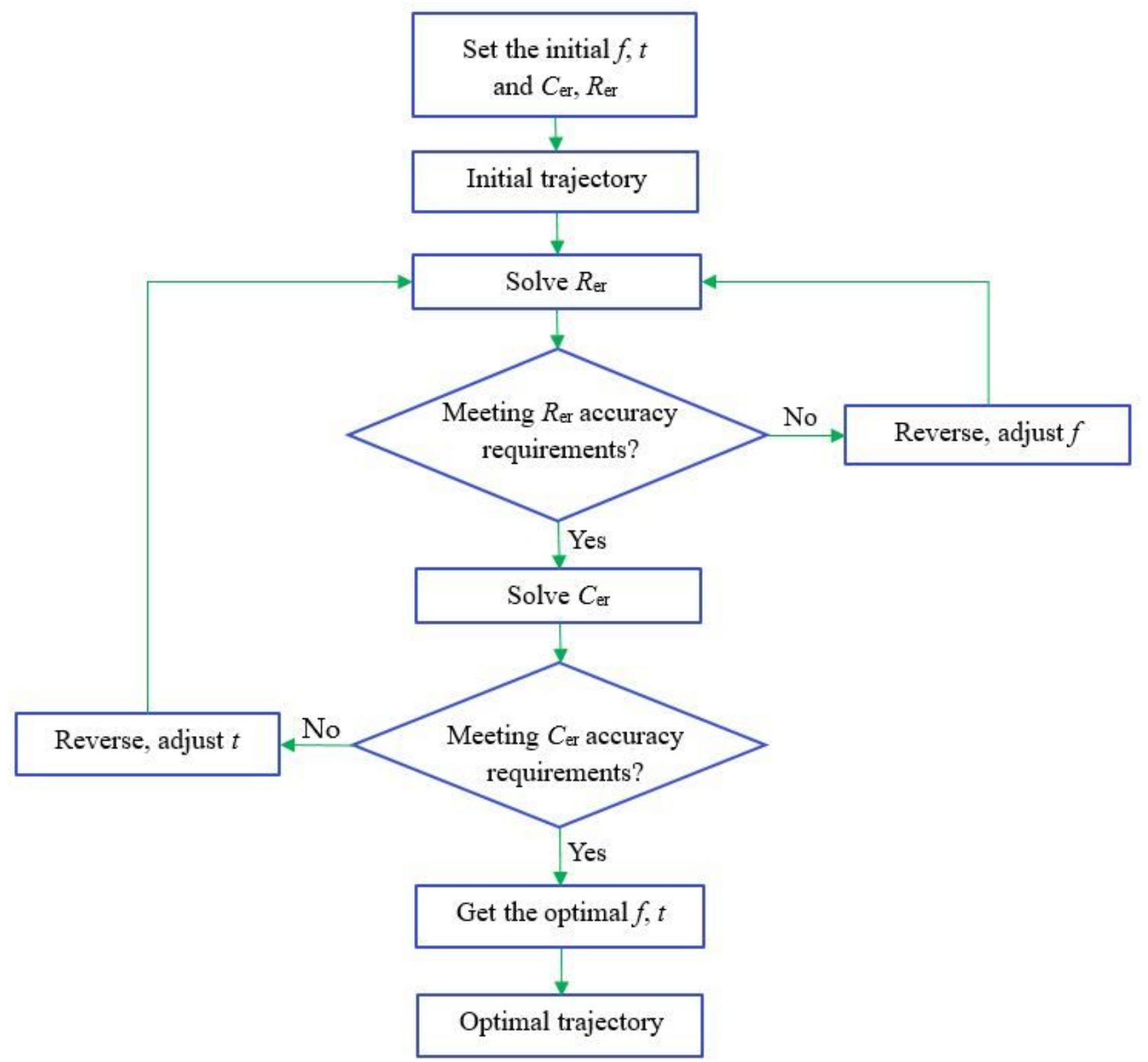

Figure 9

Schematic diagram for optimal trajectory generation 

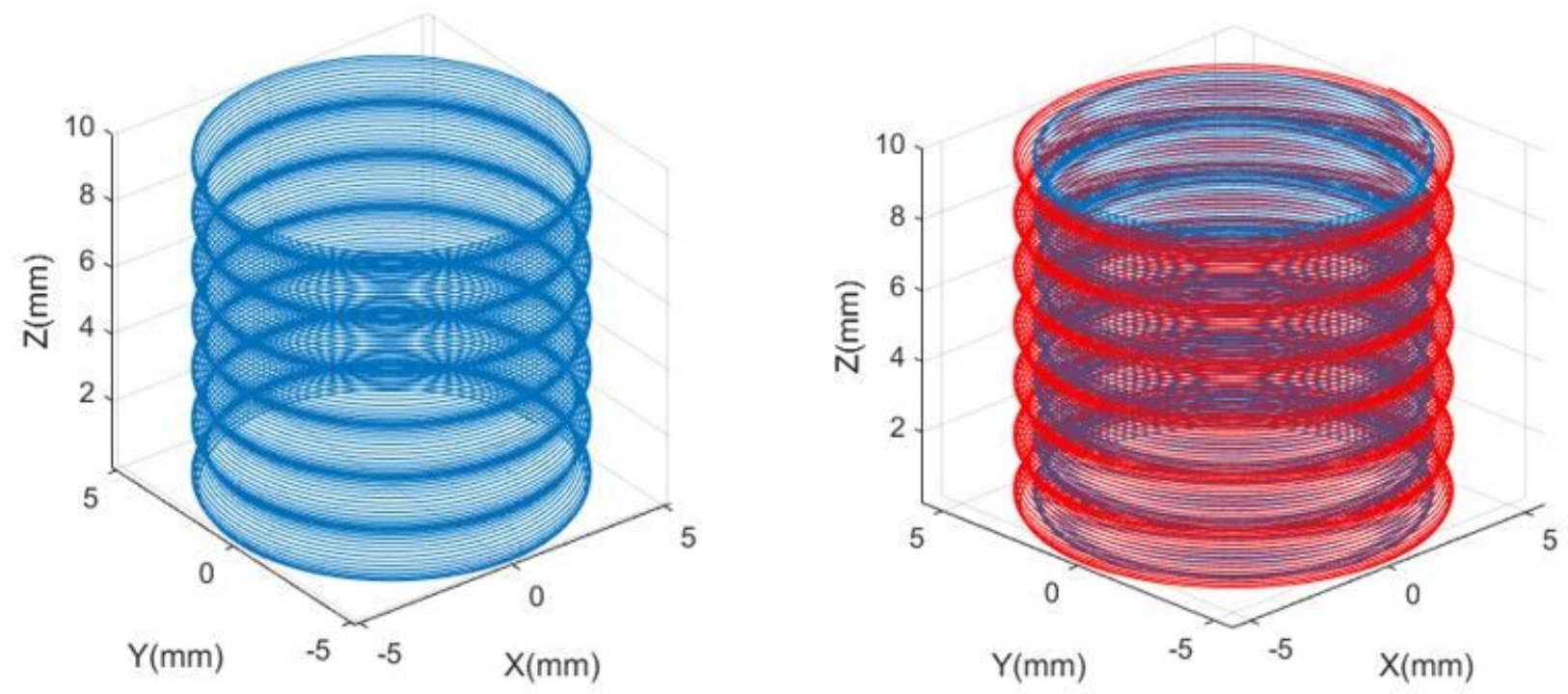

Figure 10

Blue tool contact trajectory (left) and red tool position trajectory (right) of CSWGS
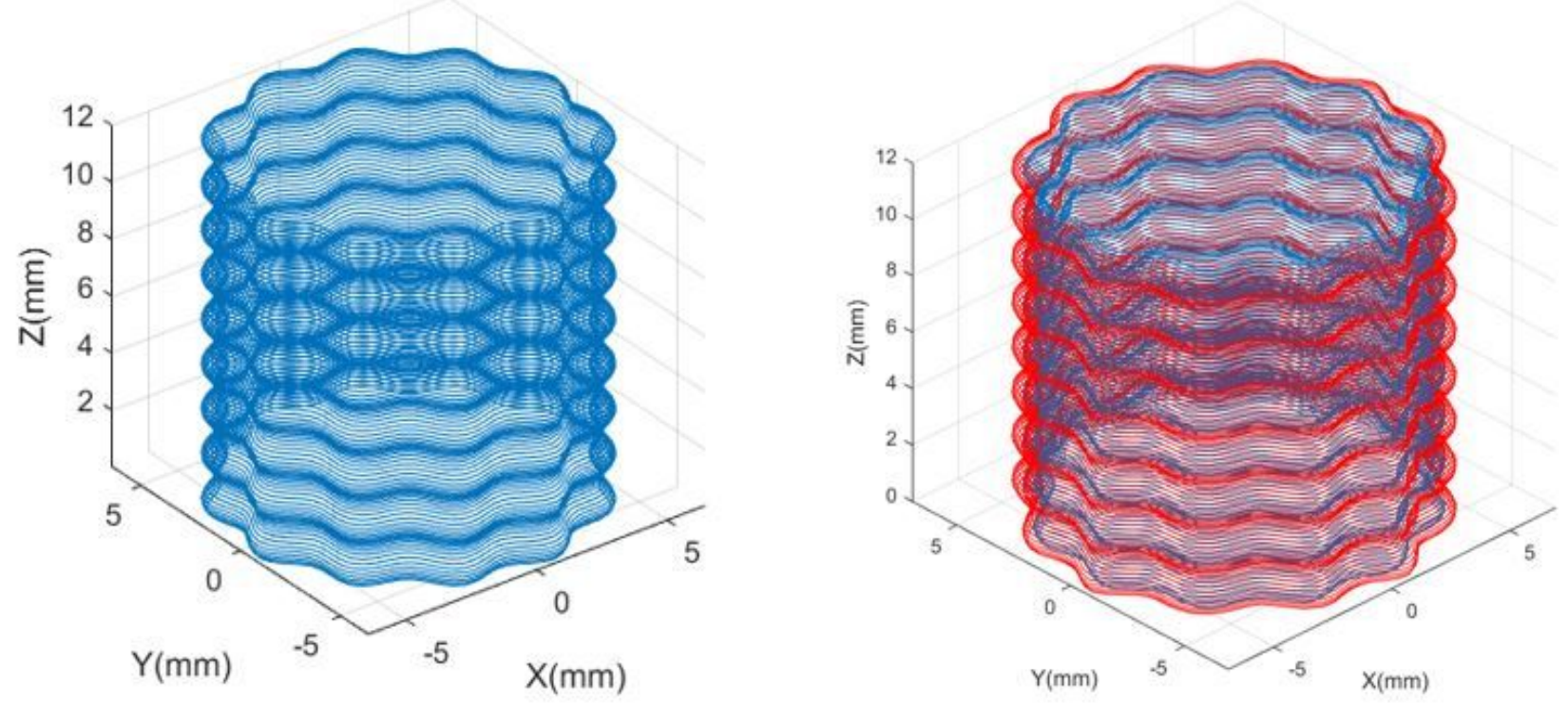

Figure 11

Blue tool contact trajectory (left) and red tool position trajectory (right) of CSMS 


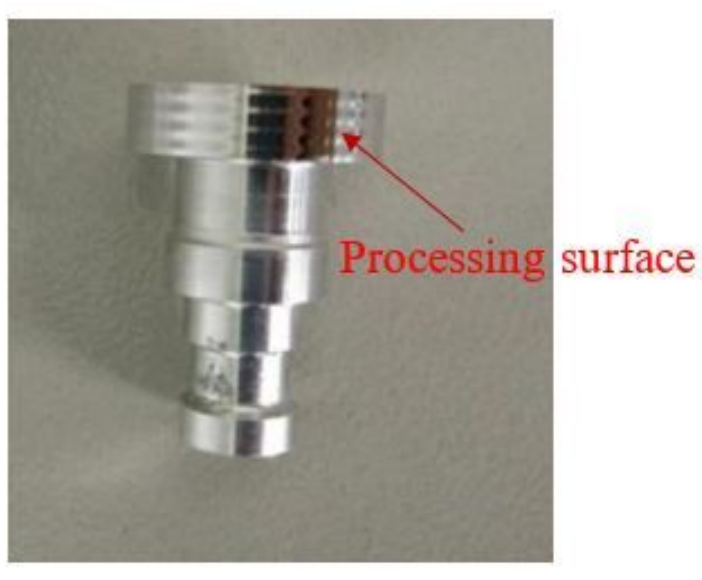

(a)

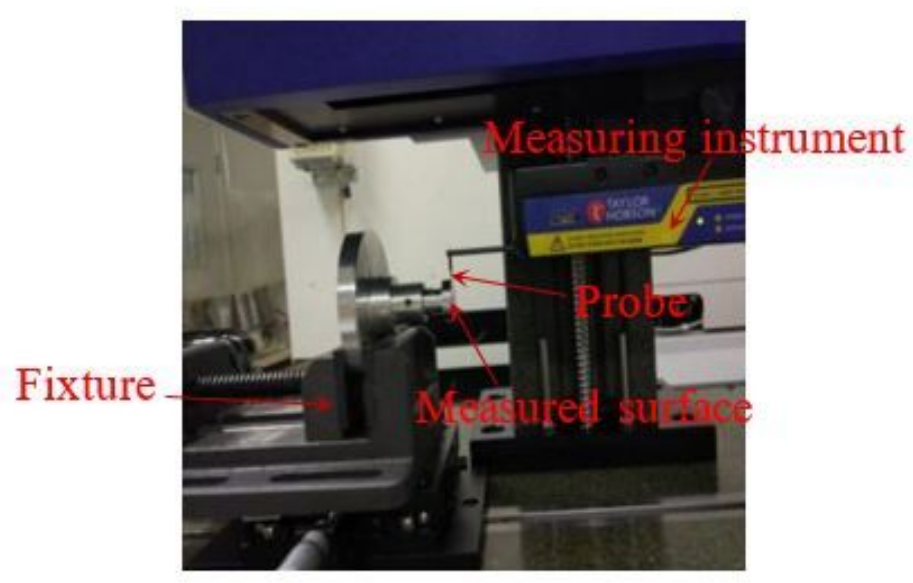

(b)

\section{Figure 12}

Final processing object and microfeature measurement of CSWGS. (a) Final object; (b) Measurement.

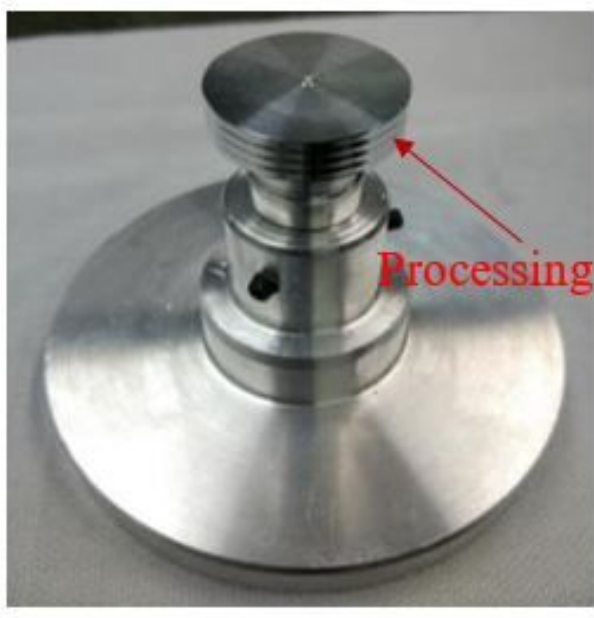

(a)

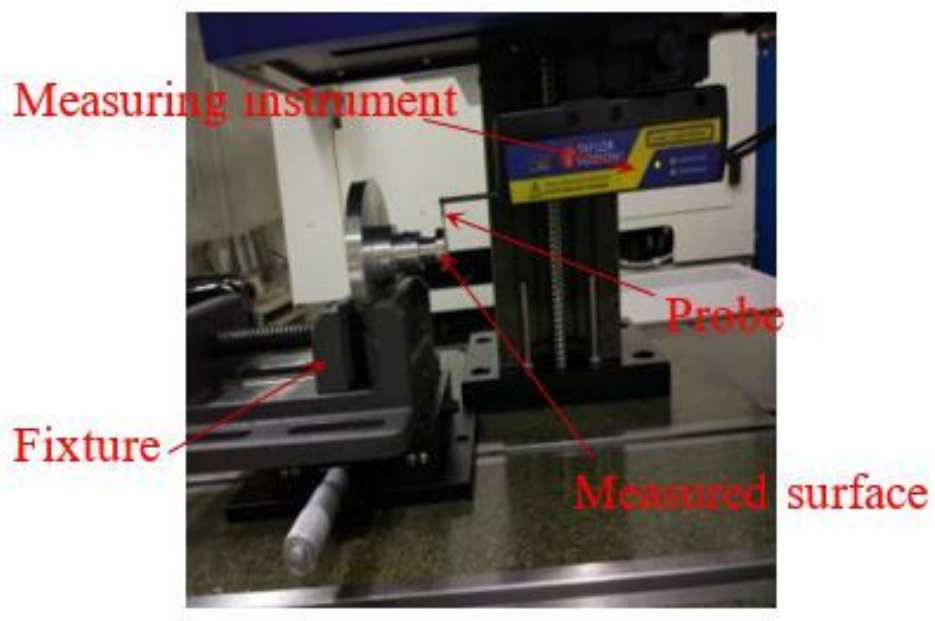

(b)

\section{Figure 13}

Final processing object and microfeature measurement of CSMS (a) Final object; (b) Measurement. 


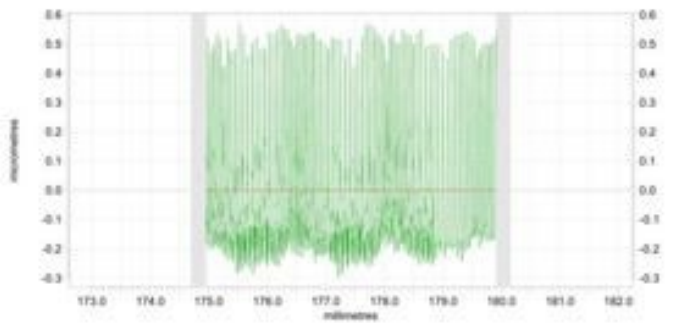

(a)

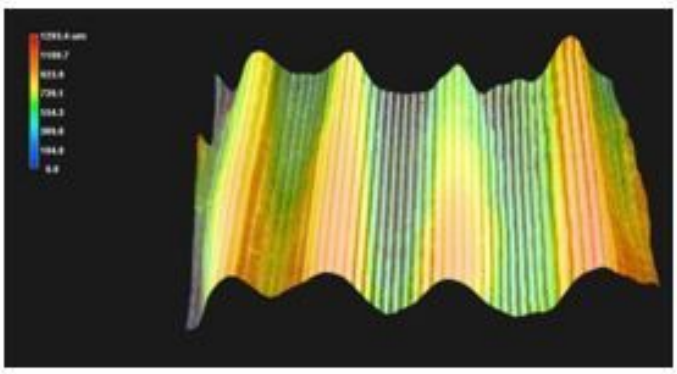

(c)

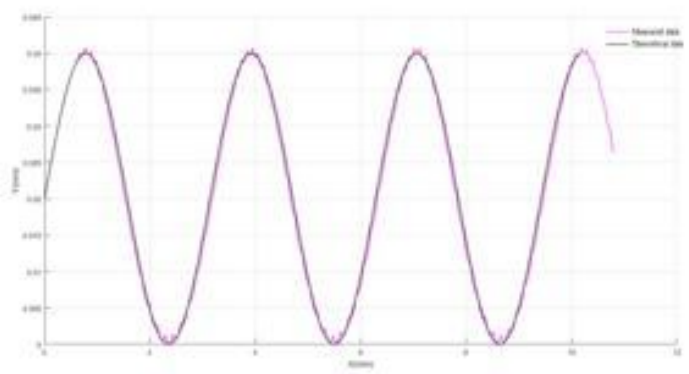

(e)

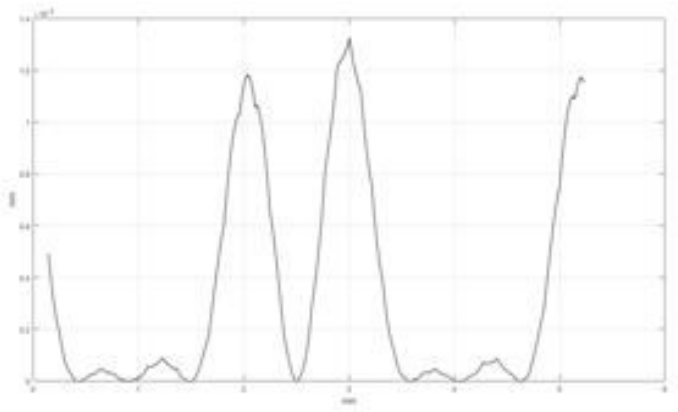

(g)

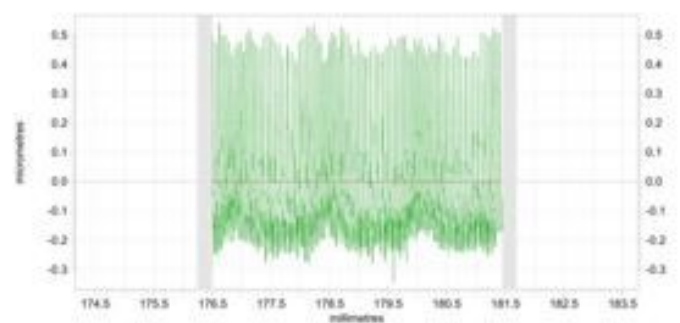

(b)

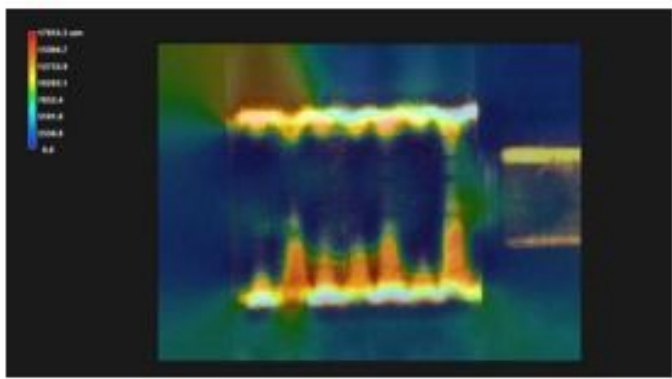

(d)

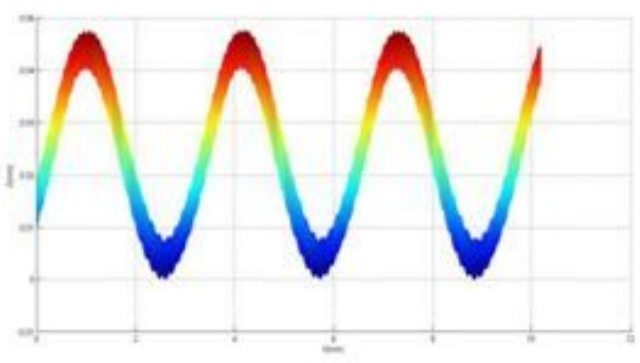

(f)

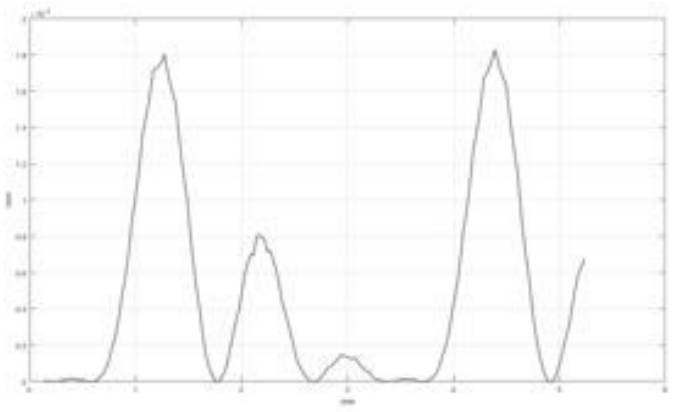

(h)

Figure 14

Measurement and calculation results of CSWGS and CSMS 